\title{
Flow and Displacement of Waxy Crude Oils in a Homogenous Porous Medium: a Numerical Study
}

\author{
A. Salehi-Shabestari ${ }^{1}$, A. Ahmadpour ${ }^{2}$, M. Raisee ${ }^{1}$, and K. Sadeghy ${ }^{*}$ \\ ${ }^{1}$ Center of Excellence in Design and Optimization of Energy Systems (CEDOES), \\ School of Mechanical Engineering, College of Engineering, University of Tehran, \\ Tehran, Iran, P.O. Box: 11155-4563, sadeghy@ut.ac.ir \\ ${ }^{2}$ Department of Mechanical Engineering, Amirkabir University of Technology, Tehran, Iran
}

\begin{abstract}
In the present study, the displacement of waxy crude oils is numerically/theoretically investigated in the water-flooding operation. The oil was assumed to obey the Houska model-a robust thixotropic fluid model which is often realized to well describe the rheology of waxy oils in different parts of the world. Based on the concept of effective viscosity, a modified version of the Darcy's law was developed for this particular fluid model in order to describe its flow through a homogenous porous medium. Use was made of numerical and theoretical methods to study the displacement of Houska fluid by water in two benchmark problems: (i) the Buckley-Leverett problem, and (ii) the five-spot problem. It was found that the yield stress of the Houska fluid being variable (i.e., shear- and time-dependent) has a retarding effect on the water breakthrough phenomenon. The breakdown-to-rebuild ratio in the Houska model was shown to play a key role in the water breakthrough phenomenon provided that it is very large. At this extreme, however, the effect was attributed mostly to the shear-thinning behavior of the Houska fluid rather than its thixotropic behavior. In fact, at sufficiently low breakdown-to-rebuild ratios (i.e., when fluid's thixotropy becomes progressively more important) it had no significant effect on the water breakthrough phenomenon. Therefore, it is concluded that in competition with shear-thinning, the thixotropic behavior of Houska fluid plays a secondary role, if any, in the water-flooding operation.
\end{abstract}

Keywords: Waxy oil, porous media, water flooding, thixotropy, Houska model, Darcy's law.

\section{Introduction}

Liquid/Liquid displacement is encountered in many branches of engineering. One can mention, for example the water flooding operation widely used in the oil industry [1]. The operation typically involves injection of water into a partially-depleted reservoir in order to direct the oil left in the rock pores towards the production well. In practice, however, due to a difference between their mobility, sooner or later, the injected water reaches the production well. An estimation of the time needed by the water to breakthrough 
is of paramount importance in the oil industry as it directly affects the oil throughput. The same is true for the sweep efficiency (i.e., the volume of the oil recovered by the water) which is important as well. Due to the difficulty of carrying out experimental studies under real reservoir conditions, theoretical and/or numerical methods are often used for their estimation. But, this is not an easy task realizing the fact that we are dealing with the flow of a complex fluid through a porous medium (i.e., the oil reservoir). A review of the literature on simulating fluid flow through porous media reveals that there are different approaches for modeling such flows [2]. One can particularly mention the promising and more accurate pore-scale and network models [2]. But, due to hardware limitations and also the uncertainties normally involved at pore-scale level, such models are still far from becoming fully-operational. In fact, the continuum approach appears to be still favored in industry and academia alike for this purpose-thanks to its simplicity and adequate accuracy [3-5]. In this approach, a porous medium is described by macroscopic properties such as porosity and permeability. The flow rate is then related to the pressure gradient through the Darcy's law. This methodology works fine for ordinary crude oils, which are known to be Newtonian. Waxy crude oils, on the other hand, are known to behave as non-Newtonian fluids at sufficiently low temperatures. The Darcy's law must be modified for such complex oils before it can be invoked in any meaningful theoretical and/or numerical study. The idea is to replace the Newtonian viscosity in this law by an appropriate non-Newtonian effective viscosity for the fluid under investigation.

The idea of effective viscosity has been shown to work well for generalized Newtonian fluids such as the Herschel-Bulkley model [6-10]. The problem is that the yield stress and viscosity of waxy crude oils is variable (i.e., shear- and time-dependent). That is to say that, waxy crude oils are thixotropic in addition to being viscoplastic [11,12]. Pearson and Tardy [13], and Pritchard and Pearson [14] appear to be the only researchers who have tried to extend the Darcy's law to thixotropic fluids. However, the thixotropic model adopted by them (namely, the modified Bautista-Manero model [15]) is not a good model for representing viscoplastic waxy crude oils. In fact, as far as we are aware, Darcy's law has not previously been extended to any rheological model representing any real waxy crude oil. This is certainly true for the Houska model [16], which is known to well describe the rheology of waxy crude oils in many parts of the world. With this in mind, in the present work we intend to first extend the classic Darcy's law to this particular fluid model and then use it for investigating its water-flooding performance in two benchmark problems: (i) the one-dimensional Buckley-Leverett problem [17], and (ii) the two-dimensional five-spot problem [18]. The main objective of the work is to see how the material parameters in the Houska model affect its breakthrough time and sweep efficiency in the water-flooding operation.

To reach its objectives, the work is organized as follows: In the next section, we formulate the mathematical framework for our fluid-mechanics problem by developing a modified Darcy's law for the 
Houska fluid. We also describe the benchmark problems mentioned above in more details together with introducing the initial and boundary conditions required to close the problem. We then proceed with describing the numerical method of solution in some details. Numerical results are presented next together with offering our understanding of their significance. The work is concluded by highlighting its major findings.

\section{Mathematical Formulation}

We consider the pressure-driven flow of a waxy oil in a porous medium of uniform permeability and porosity. Although three-dimensional models are often used in the oil sector for simulating the characteristics of real reservoirs [19,20], simplified one- and/or two-dimensional models are still in widespread use for elucidating the mechanisms involved in the recovery process. One such model, which was introduced by Buckley and Leverett in 1941 [17], tries to describe the displacement of immiscible fluids (for example, water/oil) in sand reservoirs. In their seminal paper, Buckley and Leverett [17] introduced a simplified mass-balance-equation (MBE) to model the frontal advance in the onedimensional flow through a constant-area reservoir. They used fractional flow as a function of water saturation for this purpose having assumed that the oil and water are both Newtonian fluids. To simplify the analysis, they neglected the capillary pressure. As to the initial condition, it is assumed that the reservoir is initially filled with the oil. As the boundary condition(s), it was assumed that water is being injected (with a known rate) at one end with the oil being produced (at a constant rate) at the other end. Having analytically solved the governing equations, Buckley and Leverett [17] determined the breakthrough time and sweep efficiency through determining the speed of the waterfront. Figure 1 shows a typical waterfront which is propagating from left to right in such a simplistic oil reservoir.

\section{Figure 1}

Another benchmark problem which is in common use in the oil industry is the homogenous quarter fivespot problem [18]. It is a two-dimensional water-flooding test case in which the production well is surrounded by four injection wells in a repeating pattern. Figure 2 shows the location of the injection/production wells in such an arrangement. In practice, water is injected from the injection wells and pushes the oil towards the production wells. For this benchmark problem, we rely on the water saturation profiles in order to determine the water breakthrough time.

\section{Figure 2}


The starting point for a mathematical treatment of the liquid/liquid displacement in the two benchmark problems mentioned above is the conservation of mass for each phase. For two immiscible liquids flowing simultaneously through a porous medium, the mass balance for each phase can be written as [20]:

$$
\frac{\partial}{\partial t}\left(\phi \rho_{\alpha} S_{\alpha}\right)+\nabla \cdot\left(\rho_{\alpha} \mathbf{u}_{\alpha}\right)=q_{\alpha} ; \alpha=w, o
$$

where $t$ is the time, $\phi$ is the reservoir's porosity, $\rho$ is the density of each phase, $S$ is the phase saturation, $\mathbf{u}=(\mathrm{u}, \mathrm{v})$ is the phase velocity vector, and $q$ is the rate of injection/production of each phase. The subscript $\alpha$ in the above equation refers to each phase with "w" denoting the displacing liquid (say, water) and "o" denoting the displaced liquid (say, oil). For Newtonian/Newtonian displacement, phase velocities are often calculated using the classic Darcy's law. For non-Newtonian fluids, in general, this law is not valid in its original form. For generalized Newtonian fluids (GNF), however, this law can be extended by simply replacing the Newtonian viscosity by an effective viscosity. With this in mind, for the displaced fluid we can write [20]:

$$
\mathbf{u}_{\mathrm{o}}=-\mathrm{k} \frac{\mathrm{k}_{\mathrm{ro}}}{\mu_{\mathrm{eff}, \mathrm{o}}} \nabla \mathrm{p},
$$

where $\mathrm{p}$ is the isotropic pressure, $\mathrm{k}$ is the reservoir's permeability, $\mathrm{k}_{\mathrm{ro}}$ is the relative permeability of the oil phase (which is assumed to be a function of the phase saturation, $S$ ), and $\mu_{\mathrm{eff}, \mathrm{o}}$ is the effective viscosity of the waxy oil. For the displacing Newtonian fluid, we resort to the original Darcy model; that is:

$$
\mathbf{u}_{\mathrm{w}}=-\mathrm{k} \frac{\mathrm{k}_{\mathrm{rw}}}{\mu_{\mathrm{w}}} \nabla \mathrm{p},
$$

where $\mu_{\mathrm{w}}$ is the viscosity of the displacing liquid, and $\mathrm{k}_{\mathrm{rw}}$ is the relative permeability of water. By substituting Eqs. 2 and 3 into Eq. 1, we easily obtain [20]:

$$
\begin{aligned}
& \frac{\partial}{\partial \mathrm{t}}\left(\phi \rho_{\mathrm{w}} \mathrm{S}_{\mathrm{w}}\right)-\nabla \cdot\left(\rho_{\mathrm{w}} \mathrm{k} \frac{\mathrm{k}_{\mathrm{rw}}}{\mu_{\mathrm{w}}} \nabla \mathrm{p}\right)=\mathrm{q}_{\mathrm{w}}, \\
& \frac{\partial}{\partial \mathrm{t}}\left[\phi \rho_{\mathrm{o}}\left(1-\mathrm{S}_{\mathrm{w}}\right)\right]-\nabla \cdot\left(\rho_{\mathrm{o}} \mathrm{k} \frac{\mathrm{k}_{\mathrm{ro}}}{\mu_{\text {eff }, \mathrm{o}}} \nabla \mathrm{p}\right)=\mathrm{q}_{\mathrm{o}} \cdot
\end{aligned}
$$

where $S_{o}+S_{w}=1$. It is to be noted that in extending the Darcy's law to generalized Newtonian liquids through the above relationships, it has tacitly been assumed that the pressure at the oil/water interface is identical. That is to say that, for ease of analysis, the capillary pressure has been neglected in this work. 
The above equations are the equations governing the displacement of an arbitrary non-Newtonian liquid by a Newtonian liquid. To simulate the water-flooding operation of waxy oils, the effective viscosity of the displaced oil $\left(\mu_{\text {eff, }}\right)$ needs to be known in Eq. 5. And, this necessitates having a good knowledge about the rheological model of such oils [21].

\subsection{Rheological Model}

Rheological data available for waxy oils have revealed that, regardless of their country of origin, they exhibit viscoelasticity, viscoplasticity, and thixotropy [22-26]. The latter behavior means that their yield stress and viscosity are time-dependent in addition to being shear-dependent. At present, there is no comprehensive constitutive equation valid for all kinds of waxy oils. Rheological models developed in the past have mostly focused on the viscoplastic/thixotropic aspects of such fluids. Phenomenological models such as Casson model [27], Bigham model [28], Herschel-Buckley model [29], and Richardson model [30] have been found to fit the rheological data for certain waxy oils provided that they are modified appropriately. Structural-based models do not need any kind of modification, which is perhaps why they are often preferred by the researchers. The most sophisticated of such models is the de Souza Mendes model [31], and the Dullaert-Mewis model [32]. These two thixoelastic/viscoplastic models can virtually address all aspects of their rheology. Unfortunately, they are too complicated for use in numerical/theoretical studies. Less demanding structural models such as Zhao's model [33] and/or Houska's model [16] are more appropriate for such studies.

The Zhao and Houska models are good only for ideal (i.e., inelastic) thixotropic fluids [see Ref. 34]. Although, in true sense, all waxy oils are somewhat elastic, the fact that these two rheological models can fit the viscometric data for several commercial waxy oils [35] suggests that perhaps the elasticity of these oils is overshadowed by the shear-thinning, viscoplasticity, and thixotropy. The fact is that inelastic thixotropic models are good for fundamental studies trying to figure out the role played by thixotropy on any given fluid-mechanics problem. That is, they exclude any complications which might arise through fluid's elasticity. In this regard, it should be conceded that Zhao's model better fits the rheological data for waxy oils. But, this is simply because it incorporates two distinct structural parameters. Regardless of the fact that having two structural parameters in a single model is hard to swallow, the model is too complicated for theoretical or even numerical studies. Houska model, on the other hand, enjoys having a better physical basis. Furthermore, thanks to its simplicity, it is more probable to render itself to a theoretical and/or numerical solution. The model also well represents the rheology of waxy oils from different parts of the world [35]. Indeed, our own rheological data, presented in Appendix A, shows that this model is also good for a commercial waxy crude oil produced in the Middle East. For such reasons, 
we have decided to rely on this fluid model for the present study. Further interest in this model stems from the fact the start-up flow of this particular fluid model has been the subject of much interest in recent years [36-39]. To have a complete picture, it is necessary to study the flow of the same fluid at the production sage where it has to flow through a porous reservoir (say, at the upstream sector of the oil industry). Having said this, we would like to stress that there are waxy oils which do not completely obey this rheological model $[40,41]$.

In one-dimensional flows (e.g., simple shear) the incompressible version of the Houska model reads as [16]:

$$
\begin{array}{ll}
\tau=\tau_{\mathrm{y}, 0}+\lambda\left(\tau_{\mathrm{y}, 1}-\tau_{\mathrm{y}, 0}\right)+\left[\eta_{0}+\lambda\left(\eta_{1}-\eta_{0}\right)\right] \dot{\gamma} & ; \quad|\tau| \geq \tau_{\mathrm{y}, 0}+\lambda\left(\tau_{\mathrm{y}, 1}-\tau_{\mathrm{y}, 0}\right) \\
\dot{\gamma}=0 & ; \quad|\tau|<\tau_{\mathrm{y}, 0}+\lambda\left(\tau_{\mathrm{y}, 1}-\tau_{\mathrm{y}, 0}\right)
\end{array}
$$

where, in simple shear, $\tau$ is the shear stress, and $\dot{\gamma}$ is the shear rate. In this fluid model, $\lambda$ is the structural parameter which represents the level of microstructure left in the material at each instant of time; in fact, $\lambda=1$ refers to fully-rebuilt structure, and $\lambda=0$ to completely broken-down structure. The yield stress and the viscosity corresponding to $\lambda=1$ are denoted by $\tau_{y, 1}$ and $\eta_{1}$ (the so-called zero-shear parameters). Similarly, the yield stress and viscosity corresponding to $\lambda=0$ are referred to by $\tau_{\mathrm{y}, 0}$ and $\eta_{0}$ (the socalled infinite-shear parameters)—by default, we have: $\tau_{\mathrm{y}, 1}>\tau_{\mathrm{y}, 0}$ and $\eta_{1}>\eta_{0}$. In the Houska model, the kinetic equation governing the time-evolution of the structural parameter is of the following form [16]:

$$
\frac{\mathrm{D} \lambda}{\mathrm{Dt}}=\mathrm{a}(1-\lambda)-\mathrm{b} \lambda \dot{\gamma}
$$

where "a" and "b" are material properties. With the structural parameter being time- and shear-dependent, it is obvious that the fluid's viscosity and yield stress are also simultaneously time- and shear-dependent. While the material property "a" controls the degree of structure recovery (say, through the ever-present Brownian motion), "b" controls the rate of structure destruction, which is deemed to be shear-dependent. Wachs et al. [38] argued that, for waxy oils the time scale for the structure buildup is of the order of hours whereas for the structure breakdown it is of the order of seconds. One might therefore be tempted to neglect the recovery term in Eq. 7 for the restart problem—at least, at its early stages [39]. This appears to be also the case for the flow through a porous medium, which is inherently a slow flow. Nevertheless, we have decided to keep the rebuild term in this equation no matter how small it is. In the next section, we show that the breakdown-to-rebuild ratio, b/a, strongly controls the severity of the fluid's shear-thinning behavior. In Appendix B it has been shown that the degree of fluid's thixotropy is also controlled by the same ratio. Since this ratio has the dimension of time, it can conveniently be referred to as the fluid's characteristic time-its inverse is sometimes referred to as the characteristic shear rate [see Ref. 42]. 


\subsection{Effective Viscosity of the Houska Model}

As highlighted by Pritchard and Pearson [14], the porous media community still awaits a comprehensive framework to be developed in future for describing the dynamics of thixotropic fluids in a porous medium [see, also, ref. 13]. As a short-term remedy, use can be made of the Darcy's law which can fortunately be extended to ideal thixotropic fluids (e.g., the Houska fluid) [34] through invoking the concept of an effective viscosity [43]. The only question left to be answered is how to determine the effective viscosity of the Houska fluid. And, it turns out that the well-known Poiseuille flow in a pipe can well be used for this purpose. McKinely et al [44] resorted to a simple analogy between the Darcy's law and the Poiseuille's law and showed that $\sqrt{\mathrm{k} / \phi}$ of a porous media is proportional to the pipe radius with the constant of proportionality depending on the pore size distribution and the tortuosity of the pores in the any particular porous medium. (It is worth-mentioning that Poiseuille flow is also the basis of another idea which models a porous medium by a bundle of capillary tubes of the same or different diameters [45].) To proceed with this simple idea for finding the effective viscosity of a Houska fluid, we can rely on an analytical solution recently derived in a circular pipe for this particular fluid [46]. That work, however, has ignored the Rabinowitch correction factor. To take into account this factor, we start from the Rabimowitch formula for laminar flow of a general class of fluids through a capillary having a radius of $\mathrm{R}$ and a length of L [47]; that is:

$$
\frac{\mathrm{Q} \cdot \tau_{\mathrm{w}}^{3}}{\pi \mathrm{R}^{3}}=\int_{0}^{\tau_{\mathrm{w}}} \tau^{2} \dot{\gamma} \mathrm{d} \tau
$$

where $\mathrm{Q}$ is the flow rate and $\tau_{\mathrm{w}}=(\mathrm{R} / 2)(-\partial \mathrm{p} / \partial \mathrm{x})$ is the wall shear stress. This equation presumes that the flow is fully-developed and steady. The first assumption is plausible on the account that in a typical reservoir, $\mathrm{L}$ is of the order of kilometers so that we have: $\mathrm{L}>>\mathrm{R}$. As to the second assumption, it should be noted that flow through a porous medium is inherently a slow flow. With the characteristic length (L) being very large, it means that a thixotropic oil has plenty of time to reach equilibrium as soon as it enters the capillary. From Eq. 7 the equilibrium value of the structural parameter is obtained as:

$$
\lambda_{\mathrm{ss}}=\frac{1}{1+\mathrm{b} / \mathrm{a} \dot{\gamma}}
$$

By inserting this equation into the one-dimensional form of the Houska model (see Eq. 6) we obtain,

$$
\tau=\tau_{\mathrm{y}, 0}+\frac{1}{1+\mathrm{b} / \mathrm{a} \dot{\gamma}}\left(\tau_{\mathrm{y}, 1}-\tau_{\mathrm{y}, 0}\right)+\left[\eta_{0}+\frac{1}{1+\mathrm{b} / \mathrm{a} \dot{\gamma}}\left(\eta_{1}-\eta_{0}\right)\right] \dot{\gamma} .
$$

This equation can be regarded as the steady version of the Houska model applicable to pipe flow or any other one-dimensional viscometric flow. From this equation it can be concluded a Houska fluid behaves 
like a generalized Newtonian fluid in steady shear. In fact, a plot of the apparent viscosity of the fluid [see Ref. 48] shows that, at equilibrium, a Houska fluid can be regarded as a shear-thinning fluid with the severity of its shear-thinning being controlled by the ratio b/a. Actually, the fluid becomes progressively more shear-thinning when this ratio is increased. To determine the effective viscosity of the fluid, Eq. 10 is re-written as [46],

$$
\dot{\gamma}^{2}+\left(\frac{\mathrm{a}}{\mathrm{b}} \cdot \frac{\eta_{1}}{\eta_{0}}+\frac{\tau_{\mathrm{y}, 0}-\tau}{\eta_{0}}\right) \dot{\gamma}+\frac{\mathrm{a}}{\mathrm{b}} \cdot \frac{\tau_{\mathrm{y}, 1}-\tau}{\eta_{0}}=0
$$

This second-order algebraic equation can easily be solved for $\dot{\gamma}$ and the physically-acceptable solution is determined as [46]:

$$
\dot{\gamma}=\frac{1}{2 \eta_{0}}\left\{\tau-\left(\tau_{\mathrm{y}, 0}+\frac{\mathrm{a}}{\mathrm{b}} \eta_{1}\right)+\sqrt{\tau^{2}-2\left[\frac{\mathrm{a}}{\mathrm{b}}\left(\eta_{1}-2 \eta_{0}\right)+\tau_{\mathrm{y}, 0}\right] \tau+\left(\tau_{\mathrm{y}, 0}+\frac{\mathrm{a}}{\mathrm{b}} \eta_{1}\right)^{2}-\frac{4 a}{\mathrm{~b}} \eta_{0} \tau_{\mathrm{y}, 1}}\right\} .
$$

Substituting this equation into Eq. 8 we obtain,

$$
\frac{\mathrm{Q} \cdot \tau_{\mathrm{w}}^{3}}{\pi \mathrm{R}^{3}} 2 \eta_{0}=\int_{\tau_{\mathrm{y}, 1}}^{\tau_{\mathrm{w}}} \tau^{2}\left\{\tau-\left(\tau_{\mathrm{y}, 0}+\frac{\mathrm{a}}{\mathrm{b}} \eta_{1}\right)+\sqrt{\tau^{2}-2\left[\frac{\mathrm{a}}{\mathrm{b}}\left(\eta_{1}-2 \eta_{0}\right)+\tau_{\mathrm{y}, 0}\right] \tau+\left(\tau_{\mathrm{y}, 0}+\frac{\mathrm{a}}{\mathrm{b}} \eta_{1}\right)^{2}-\frac{4 a}{\mathrm{~b}} \eta_{0} \tau_{\mathrm{y}, 1}}\right\} d \tau .
$$

This equation can be rewritten in terms of the superficial velocity, u:

$$
\mathrm{u}=\frac{\mathrm{R} \phi}{2 \eta_{0} \tau_{\mathrm{w}}^{3}} \int_{\tau_{\mathrm{y}, 1}}^{\tau_{\mathrm{w}}} \tau^{2}\left\{\tau-\left(\tau_{\mathrm{y}, 0}+\frac{\mathrm{a}}{\mathrm{b}} \eta_{1}\right)+\sqrt{\tau^{2}-2\left[\frac{\mathrm{a}}{\mathrm{b}}\left(\eta_{1}-2 \eta_{0}\right)+\tau_{\mathrm{y}, 0}\right] \tau+\left(\tau_{\mathrm{y}, 0}+\frac{\mathrm{a}}{\mathrm{b}} \eta_{1}\right)^{2}-\frac{4 \mathrm{a}}{\mathrm{b}} \eta_{0} \tau_{\mathrm{y}, 1}}\right\} \mathrm{d} \tau,
$$

In an analogy with the classic Darcy's law we have,

$$
\mathrm{u}=\frac{\mathrm{k}}{\mu_{\mathrm{eff}, \mathrm{o}}}\left(-\frac{\partial \mathrm{p}}{\partial \mathrm{x}}\right)
$$

where $\mathrm{k}$ is the permeability of the porous medium so that we have,

$$
\mathrm{k}=\frac{\mathrm{R}^{2} \phi}{8}
$$

By comparing Eq. 14 with Eqs. 15-16, the effective viscosity of the Houska oil, $\mu_{\text {eff }, o}$, is obtained as:

$$
\frac{1}{\mu_{\mathrm{eff}, 0}}=\frac{2}{\eta_{0} \tau_{\mathrm{w}}^{4}} \int_{\tau_{\mathrm{y}, 1}}^{\tau_{\mathrm{w}}} \tau^{2}\left\{\tau-\left(\tau_{\mathrm{y}, 0}+\frac{\mathrm{a}}{\mathrm{b}} \eta_{1}\right)+\sqrt{\tau^{2}-2\left[\frac{\mathrm{a}}{\mathrm{b}}\left(\eta_{1}-2 \eta_{0}\right)+\tau_{\mathrm{y}, 0}\right] \tau+\left(\tau_{\mathrm{y}, 0}+\frac{\mathrm{a}}{\mathrm{b}} \eta_{1}\right)^{2}-\frac{4 \mathrm{a}}{\mathrm{b}} \eta_{0} \tau_{\mathrm{y}, 1}}\right\} \mathrm{d} \tau
$$

The integral term on the right-hand-side of this equation can be symbolically determined using the Maple software. The effective viscosity of the Houska fluid then becomes [48]: 
$\frac{1}{\mu_{\text {eff }, 0}}=\frac{1}{2 \eta_{0}}\left[1-\left(\frac{\tau_{y, 1}}{\tau_{w}}\right)^{4}\right]-\frac{2}{3 \eta_{0} \tau_{w}}\left(\tau_{y, 0}+\frac{a}{b} \eta_{1}\right)\left[1-\left(\frac{\tau_{y, 1}}{\tau_{w}}\right)^{3}\right]+\frac{2}{\eta_{0} \tau_{w}^{4}}\left[F\left(\tau_{w}\right)-F\left(\tau_{y, 1}\right)\right]$.

In this equation, we have:

$$
\begin{aligned}
& \mathrm{F}(\tau)=\frac{1}{24}\left(18 \mathrm{~B}^{2} \mathrm{C}-15 \mathrm{~B}^{4}-3 \mathrm{C}^{2}\right) \ln \left(\tau-\mathrm{B}+\sqrt{\tau^{2}-2 \mathrm{~B} \tau+\mathrm{C}}\right)+\frac{1}{24}(6 \tau+10 \mathrm{~B})\left(\tau^{2}-2 \mathrm{~B} \tau+\mathrm{C}\right)^{3 / 2}- \\
& \frac{5}{8}\left(\mathrm{~B}^{2}-\frac{1}{5} \mathrm{C}\right)(\mathrm{B}-\tau) \sqrt{\tau^{2}-2 \mathrm{~B} \tau+\mathrm{C}}
\end{aligned}
$$

where,

$$
B=\left[\frac{a}{b}\left(\eta_{1}-2 \eta_{0}\right)+\tau_{y, 0}\right] \quad ; \quad C=\left(\tau_{y, 0}+\frac{a}{b} \eta_{1}\right)^{2}-\frac{4 a}{b} \eta_{0} \tau_{y, 1} .
$$

To see if Eq. 18 can recover the Newtonian viscosity, we set: $\mathrm{b} / \mathrm{a}=\infty, \tau_{\mathrm{y}, 1}=\tau_{\mathrm{y}, 0}=0$, and $\eta_{1}=\eta_{0}=\mu_{\mathrm{o}}$, where $\mu_{\mathrm{o}}$ is the viscosity of the corresponding Newtonian fluid. Equation 18 then becomes:

$$
\frac{1}{\mu_{\mathrm{eff}, \mathrm{o}}}=\frac{1}{2 \mu_{\mathrm{o}}}+\frac{2}{\mu_{\mathrm{o}} \tau_{\mathrm{w}}^{4}}\left[\mathrm{~F}\left(\tau_{\mathrm{w}}\right)-\mathrm{F}(0)\right]
$$

On the other hand, for Newtonian fluids, B and C (see Eq. 19b) are both equal to zero. Therefore, from Eq. 19a, F( $\tau)$ can be obtained as:

$$
\mathrm{F}(\tau)=\frac{1}{24}(6 \tau)\left(\tau^{2}\right)^{3 / 2}=\frac{\tau^{4}}{4}
$$

According to Eq. 20a the effective viscosity of a Newtonian fluid becomes:

$$
\frac{1}{\mu_{\mathrm{eff}, \mathrm{o}}}=\frac{1}{2 \mu_{0}}+\frac{2}{\mu_{0} \tau_{\mathrm{w}}^{4}} \frac{\tau_{\mathrm{w}}^{4}}{4}=\frac{1}{2 \mu_{0}}+\frac{1}{2 \mu_{0}}=\frac{1}{\mu_{0}} \Rightarrow \mu_{\mathrm{eff}, \mathrm{o}}=\mu_{0}
$$

In other words, for Newtonian fluids the above formulation is reduced to the classic Darcy's law, as expected. Now, before proceeding any further, we try to make all pertinent parameters dimensionless. To that end, we substitute [see Ref. 13]:

$$
\mathrm{x}^{*}, \mathrm{y}^{*}=\frac{\mathrm{x}, \mathrm{y}}{\mathrm{L}} ; \mathrm{t}^{*}=\frac{\mathrm{t}}{\Gamma / \mathrm{Q}_{\mathrm{w}, \mathrm{in}}} ; \mu^{*}=\frac{\mu}{\eta_{1}} ; \mathrm{u}^{*}, \mathrm{v}^{*}=\frac{\mathrm{u}, \mathrm{v}}{\mathrm{Q}_{\mathrm{w}, \mathrm{in}} \mathrm{L} / \Gamma}, \mathrm{p}^{*}=\frac{\mathrm{p}}{\eta_{1} \mathrm{Q}_{\mathrm{w}, \mathrm{in}} \mathrm{L}^{2} / \Gamma \mathrm{k}} ; \tau^{*}=\frac{\tau}{\eta_{1} \mathrm{Q}_{\mathrm{w}, \mathrm{in}} \mathrm{L} / \Gamma \mathrm{k}^{1 / 2}}
$$

where $\Gamma$ is the total volume of the domain, $\mathrm{x}, \mathrm{y}$ are the local coordinates, $\mathrm{u}, \mathrm{v}$ are the (Darcy) velocity components, and $\mathrm{Q}_{\mathrm{w}, \mathrm{in}}$ is the flow rate of the injected water. It is to be noted that, as suggested in Ref. 13, we have relied on $\mathrm{Q}_{\mathrm{w}, \mathrm{in}} \mathrm{L} / \Gamma$ as the characteristic (Darcy) velocity, on $\Gamma / \mathrm{Q}_{\mathrm{w}, \text { in }}$ as the characteristic time, and on $\mathrm{Q}_{\mathrm{w}, \mathrm{in}} \mathrm{L} / \Gamma \mathrm{k}^{1 / 2}$ as the characteristic shear rate. (By so-doing, all terms in the governing equations become of the same order.) Having substituted the above dimensionless parameters into Eqs. 4-5 and 18, 
the dimensionless form of the equations governing the water-flooding operation of a Houska fluid can easily be obtained. Having dropped the asterisks " **” above dimensionless parameters for convenience, we then obtain [48],

$$
\begin{aligned}
& \frac{\partial}{\partial \mathrm{t}}\left(\phi \mathrm{S}_{\mathrm{w}}\right)-\nabla \cdot\left(\frac{\mathrm{k}_{\mathrm{rw}}}{\mu_{\mathrm{w}}} \nabla \mathrm{p}\right)=\mathrm{q}_{\mathrm{w}}, \\
& \frac{\partial}{\partial \mathrm{t}}\left[\phi\left(1-\mathrm{S}_{\mathrm{w}}\right)\right]-\nabla \cdot\left(\frac{\mathrm{k}_{\mathrm{ro}}}{\mu_{\mathrm{eff}, \mathrm{o}}} \nabla \mathrm{p}\right)=\mathrm{q}_{\mathrm{o}}, \\
& \mu_{\mathrm{eff}, \mathrm{o}}=\left\{\frac{1}{2 \mathrm{M}}\left[1-\left(\frac{\tau_{\mathrm{y}, 1}}{\tau_{\mathrm{w}}}\right)^{4}\right]-\frac{2}{3 \mathrm{M} \tau_{\mathrm{w}}}\left(\tau_{\mathrm{y}, 0}+\frac{1}{\mathrm{Tx}}\right)\left[1-\left(\frac{\tau_{\mathrm{y}, 1}}{\tau_{\mathrm{w}}}\right)^{3}\right]+\frac{2}{\mathrm{M} \tau_{\mathrm{w}}^{4}}\left[\mathrm{~F}\left(\tau_{\mathrm{w}}\right)-\mathrm{F}\left(\tau_{\mathrm{y}, 1}\right)\right]\right\}^{-1},
\end{aligned}
$$

where,

$$
\begin{aligned}
& F(\tau)=\frac{1}{24}\left(18 B^{2} C-15 B^{4}-3 C^{2}\right) \ln \left(\tau-B+\sqrt{\tau^{2}-2 B \tau+C}\right)+ \\
& \frac{1}{24}(6 \tau+10 B)\left(\tau^{2}-2 B \tau+C\right)^{3 / 2}-\frac{5}{8}\left(B^{2}-\frac{1}{5} C\right)(B-\tau) \sqrt{\tau^{2}-2 B \tau+C}
\end{aligned}
$$

and,

$$
\mathrm{B}=\tau_{\mathrm{y}, 0}\left[\frac{(1-2 \mathrm{M})}{\mathrm{Tx} \tau_{\mathrm{y}, 0}}+1\right] ; \quad \mathrm{C}=\left(\tau_{\mathrm{y}, 0}\right)^{2}\left[\left(1+\frac{1}{\mathrm{Tx} \tau_{\mathrm{y}, 0}}\right)^{2}-\frac{4}{\mathrm{Tx}} \mathrm{M} \frac{\tau_{\mathrm{y}, 1}}{\tau_{\mathrm{y}, 0}}\right]
$$

where $\mathrm{M}=\eta_{0} / \eta_{1}$ is the viscosity ratio, and $\mathrm{Tx}=\mathrm{b} / \mathrm{a}$ is the dimensionless breakdown-to-rebuild ratio, which is referred to as the thixotropy number in the rest of the work.

Figure 3 shows the effect of different parameters on the effective viscosity of the Houska fluid. In this figure $\Delta \mathrm{G}=\mathrm{G}_{1}-\mathrm{G}_{0}$ (where $\mathrm{G}_{0}=\tau_{\mathrm{y}, 0} / \mathrm{k}^{1 / 2}$ and $\mathrm{G}_{1}=\tau_{\mathrm{y}, 1} / \mathrm{k}^{1 / 2}$ ) is a measure of the role played by the fluid's two yield stress parameters. The shear-thinning behavior of the Houska fluid, as mentioned above, is also evident in this figure. This figure shows that at sufficiently high shear stresses (or, equivalently, high shear rates) the effective viscosity of the fluid approaches its infinite-shear asymptotic value. At low shear stresses, however, no such an asymptotic value can be seen, which is due to the fluid's yielding behavior. Figure $3 a$ shows that by an increase in the Tx number the effective viscosity is decreased. This is not surprising because by an increase in Tx, structure breakdown is promoted. Figure $3 \mathrm{~b}$ shows that, as expected, the effective viscosity increases by an increase in the viscosity ratio, M. The effect of the yield 
stress parameter, $\Delta \mathrm{G}$, on the flow curve is more subtle than others. That is, while the zero-shear viscosity is dramatically affected by $\Delta \mathrm{G}$, it virtually has no significant effect on the infinite-shear viscosity (see Fig. 3c).

\section{Figure 3}

\section{Results and Discussions}

Having modified the Darcy's law to become applicable for the Houska fluids, we can now proceed with solving Eqs. 4 and 5 for the two benchmark problems mentioned above. We are particularly interested in studying the effect of the thixotropy number (Tx), the viscosity ratio (M), and the yield stress parameter ( $\Delta \mathrm{G})$ on the breakthrough time and the sweep performance of the water-flooding operation. We have been fortunate enough to find a semi-analytical, closed-form, graphical solution for the one-dimensional Buckley-Leverett problem for the Houska model. For the five-spot problem, however, no such a solution was in close sight, and so we have resorted to numerical means for this purpose.

\subsection{The Buckley-Leverett Problem}

In an inspiring work, $\mathrm{Wu}$ and Pruess [49] found a general solution for the Buckley-Leverett problem which is applicable to generalized Newtonian fluids. Their solution shows that for such fluids, the timeevolution of the water saturation, $S_{\mathrm{w}}$, can be obtained as:

$$
\left\{\begin{array}{lr}
S_{w}=S_{w}^{\text {ult. }} & 0 \leq x \leq x_{1} \\
x=\frac{\int_{0}^{t} Q(\tau) d \tau}{\phi}\left(\frac{d f_{w}}{d S_{w}}\right) & x_{1}<x \leq x_{2} \\
S_{w}=0 & x>x_{2}
\end{array}\right.
$$

where $S_{\mathrm{w}}^{\text {ult. }}$ is the ultimate water saturation at the inlet section, $\mathrm{Q}$ is the volumetric flow rate (normalized by $\mathrm{Q}_{\mathrm{w} \text {,in }}$ as the characteristic volumetric flow), $\mathrm{f}_{\mathrm{w}}$ is the fractional flow of the water phase (defined as $\left.\mathrm{f}_{\mathrm{w}}=\mathrm{Q}_{\mathrm{w}} / \mathrm{Q}\right)$, and $\mathrm{x}_{1}$ and $\mathrm{x}_{2}$ are the two critical points. From Eq. 25, the critical points can be written as:

$$
\mathrm{x}_{1}=\frac{\int_{0}^{\mathrm{t}} \mathrm{Q}(\tau) \mathrm{d} \tau}{\phi}\left(\frac{\mathrm{df}_{\mathrm{w}}}{\mathrm{dS}_{\mathrm{w}}}\right)_{\mathrm{S}_{\mathrm{w}}=\mathrm{S}_{\mathrm{w}}^{\text {ult. }}} ; \quad \mathrm{x}_{2}=\frac{\int_{0}^{\mathrm{t}} \mathrm{Q}(\tau) \mathrm{d} \tau}{\phi}\left(\frac{\mathrm{df}_{\mathrm{w}}}{\mathrm{dS}_{\mathrm{w}}}\right)_{\mathrm{S}_{\mathrm{w}}=\mathrm{S}_{\mathrm{w} 2}} .
$$

The second critical point, $\mathrm{x}_{2}$, can be related to the advection front as:

$$
\frac{f_{w 2}}{S_{w 2}}=\left(\frac{d f_{w}}{d S_{w}}\right)_{S_{w}=S_{w 2}}
$$


To solve Eqs. 25-27 for the Houska fluid, we have found it convenient to rely on the graphical method developed by Welge [50]. The whole procedure is summarized as follows:

1) Calculate $-\left(\frac{\partial p}{\partial x}\right)$ as a function of $S_{w}$ for $0<S_{w}<1-S_{\text {or }}$ from Eqs. 28-29. Since the effective viscosity is a function of the pressure gradient, an iterative procedure is needed for this purpose; that is:

$$
\mathrm{u}_{\mathrm{w}}+\mathrm{u}_{\mathrm{o}}=-\left(\frac{\partial \mathrm{p}}{\partial \mathrm{x}}\right)\left(\frac{\mathrm{k}_{\mathrm{rw}}}{\mu_{\mathrm{w}}}+\frac{\mathrm{k}_{\mathrm{ro}}}{\mu_{\mathrm{eff}, \mathrm{o}}}\right)=1 \Rightarrow-\left(\frac{\partial \mathrm{p}}{\partial \mathrm{x}}\right)=\left[\frac{\mathrm{k}_{\mathrm{rw}}}{\mu_{\mathrm{w}}}+\frac{\mathrm{k}_{\mathrm{ro}}}{\mu_{\mathrm{eff}, \mathrm{o}}}\right]^{-1}
$$

Note that based on the characteristic Darcy velocity $\left(\mathrm{Q}_{\mathrm{w}, \mathrm{in}} \mathrm{L} / \Gamma\right)$, we have concluded that $\mathrm{u}_{\mathrm{w}}+\mathrm{u}_{\mathrm{o}}=1$. Also, the relative permeability in this equation are set at [1],

$\mathrm{k}_{\mathrm{rw}}=0.75\left(\frac{\mathrm{S}_{\mathrm{w}}}{1-\mathrm{S}_{\text {or }}}\right)^{2} ; \quad \mathrm{k}_{\mathrm{ro}}=0.75\left(1-\frac{\mathrm{S}_{\mathrm{w}}}{1-\mathrm{S}_{\mathrm{or}}}\right)^{2}$

where $S_{\text {or }}$ is the residual saturation of the oil.

2) Compute the ultimate water saturation at the inlet (i.e., where there is no oil flow) as:

$$
\left\{\begin{array}{l}
1=\frac{\left(\mathrm{k}_{\mathrm{rw}}\right)^{\max }}{\mu_{\mathrm{w}}}\left(-\frac{\partial \mathrm{p}}{\partial \mathrm{x}}\right)_{\mathrm{x}=0} \Rightarrow\left(\mathrm{k}_{\mathrm{rw}}\right)^{\mathrm{max}}=0.75\left(\frac{\mathrm{S}_{\mathrm{w}}^{\text {ult. }}}{1-\mathrm{S}_{\mathrm{or}}}\right)^{2}=\frac{\mu_{\mathrm{w}}}{\mathrm{G}_{1}} \Rightarrow \mathrm{S}_{\mathrm{w}}^{\text {ult. }}=\left(1-\mathrm{S}_{\mathrm{or}}\right) \sqrt{\frac{4 \mu_{\mathrm{w}}}{3 \mathrm{G}_{1}}} \\
\left(-\frac{\partial \mathrm{p}}{\partial \mathrm{x}}\right)_{\mathrm{x}=0}=\mathrm{G}_{1}
\end{array}\right.
$$

Note that the ultimate saturation occurs where the pressure gradient approaches its minimum value. This minimum is at $\mathrm{x}=0$ where there is no oil flow. Based on Eq. 18 and the definition of $\mathrm{G}_{1}$, the minimum pressure gradient is equal to $\mathrm{G}_{1}$. At this pressure gradient, the effective viscosity of the oil becomes equal to infinity, and so there will be no oil flow-which is why in Eq. 30 we have replaced $\left(-\frac{\partial p}{\partial x}\right)_{x=0}$ with $G_{1}$. Based on the concept of the residual-oil-saturation, one can conclude that $S_{w}$ is limited to $\left(1-S_{\text {or }}\right)$ for all points in the domain and at any given time. Therefore, for $\sqrt{4 \mu_{w} / 3 G_{1}}>1$ we can write $S_{w}^{\text {ult. }}=\left(1-S_{\text {or }}\right)$.

3) Calculate the fractional flow curve for $0<S_{w}<1-S_{\text {or }}$ from the following equation:

$$
\mathrm{f}_{\mathrm{w}}=\mathrm{u}_{\mathrm{w}}=\frac{1}{1+\frac{\mathrm{k}_{\mathrm{ro}}}{\mathrm{k}_{\mathrm{rw}}}\left(\frac{\mu_{\mathrm{w}}}{\mu_{\text {eff,o }}}\right)}
$$


4) Find the water saturation at the advection front from Eq. 27 using the graphical method developed by Weldge [50].

5) Solve Eq. 25 to obtain a complete solution at any cross-section $x$ in the range of $x_{1}<x \leq x_{2}$.

Although the main purpose of solving the Buckley-Leverette problem was to verify the code developed in this work for tackling the five-spot problem (see next section), the above solution is new for Houska fluid and therefore it merits its own right. As such, we proceed with using this solution for exploring the effect of the Houska model parameters on its displacement behavior in the water flooding operation. To that end, we typically set the parameters of the Houska fluid at (see Appendix A): $G_{0}=0.2, G_{1}=0.25$, $\mathrm{Tx}=0.1$, and $\mathrm{M}=0.8$ [48]. The other parameters pertinent to the problem are set at [1]: $\phi=0.2, \mathrm{~S}_{\mathrm{wr}}=0.0, \mathrm{~S}_{\mathrm{or}}=0.2, \mu_{\mathrm{w}}=0.01, \mathrm{k}_{\mathrm{rw}}=0.75\left(\mathrm{~S}_{\mathrm{w}} /\left(1-\mathrm{S}_{\mathrm{or}}\right)\right)$ and $\mathrm{k}_{\mathrm{rw}}=0.75\left(1-\mathrm{S}_{\mathrm{w}} /\left(1-\mathrm{S}_{\text {or }}\right)\right)$.

Figure 4 shows the effect of the thixotropic number, Tx, on the displacement of a Houska fluid in the Buckley-Leverett problem. As can be seen in Fig. 4a, by an increase in Tx number the speed of the waterfront is decreased. This means that the breakthrough time is increased by an increase in the Tx number. This is not surprising realizing the fact that by an increase in the Tx number the effective viscosity of the oil is decreased (see Fig. 3). A drop in the fluid's viscosity should translate itself into a drop in the pressure at each cross-section, as can be seen in Fig. 4b. An increase in Tx number also gives rise to an increase in the level of water saturation near the inlet section (see Fig. 4a) inferring that more oil is mobilized when this parameter is increased. That is to say that, the volume the oil swept by water is expected to increase by an increase in the Tx number, as can be seen in Fig. 4c. The effect is seen to be more noticeable at intermediate times. As a matter of fact, it saturates at sufficiently long times regardless of the Tx number.

\section{Figure 4}

The above results are valid for a Houska model, and because this rheological model represents waxy crude oils in many parts of the world, they are certainly of technological impact. On a more fundamental basis, however, the above results have the drawback that we do not know to what extent the predicted effects are due to the fluid's variable yield stress. To figure out this, we start by dropping the yield stress term from the Houska model by simply setting $G_{0}=0, G_{1}=0$ in the formulations. The reduced model, which is called the "Moore model" [51] does not fit rheological data for any waxy oil, but this is none of our concerns. What concerns us most is the notion that this model can elucidate the role played by a Houska fluid's yield stress. That is, by simply comparing the two sets of results, any probable difference can be attributed to the yield stress of the Houska model. Figure 5 shows the effect of the thixotropy 
number, Tx, on the water saturation profile and also the sweep efficiency for a Moore fluid. This figure shows that, the speed of waterfront is decreased when the Tx number is increased-as is the case for the Houska fluid (see Fig. 3). However, a comparison between the results in Fig. 4a with those in Fig. 5a reveals that the speed of the waterfront is larger for the Houska fluid as compared with the Moore fluid. Since the viscous behavior is the same for both models, one can conclude that the variable yield stress of the Houska fluid accelerates the water breakthrough phenomenon and thereby lowers the sweep efficiency. Another look at Fig. 4a and Fig. 5a shows that the level of water saturation at the inlet section of the domain is lower for the Houska fluid. This means that the yield stress of a Houska fluid lowers the volume of the oil recovered through water injection-a behavior quite similar to that found for the Bingham fluid; see Ref. 7. This notion can better be seen in Fig. 5b which shows that more oil can be recovered for the Moore fluid as compared with the Houska fluid. Moreover, the volume of the recovered oil does not exhibit an asymptotic value for the Moore fluid as is the case for the Houska fluid (cf. Fig. 4c). These findings can better be seen in Fig. 6 whch compares the response of the two fluid models in Buckley-Levertt problem.

\section{Figure 5}

\section{Figure 6}

Based on the results presented in Figs. 4-6 it is evident that the variable yield stress in the Houska model plays a significant (albeit admittedly a negative) role in the water-flooding operation. The only question remains to be answered is to what extent thixotropy is involved here. The fact that waxy oils exhibit thixotropic behavior in rheological measurements does not guarantee that their thixotropy is important in water-flooding operation. For thixotropic effects to be important in practical situations, the ratio between the characteristic time of the flow and the characteristic time of the fluid (i.e., the time needed by the fluid for its equilibrium viscosity to establish itself) should be of order one or larger [13]. If this ratio is small, thixotropic effects will not be of any significant importance; see also Ref. 52. Based on the data available in the literature for waxy oils and real reservoirs, this ratio appears not to be so large [41]. But, we will see if thixotrop is indeed influential or not in our fluid mechanics problem. To that end, it suffices to obtain new results for the Houska model for the case of $M=1$ (which ensures that its viscosity is constant) while the Tx number is being varied. By so-doing we screen out the complicating effects of a variable shear viscosity from the analysis. We then proceed with obtaining results for $\mathrm{Tx}=0$ which corresponds to the Bingham fluid. A comparison between the results obtained for $\mathrm{Tx}=0$ and results obtained for $\mathrm{Tx} \neq 0$ can then highlight the importance of thixotopic effect present in the fluid's yield 
stress. Figure 7 shows results obtained this way for the Houska model. As can be seen in this figure, at sufficiently small Tx numbers (i.e., where the fluid's thixotropy should, technically-speaking, become comparatively more important; see Appendix B) there is not much difference between the two sets of results (cf. Tx $=0$ with $\mathrm{Tx}=0.1$ ). At large Tx numbers, however, there is a marked difference between the Bingham results and the Houska results; that is, in a Bingham oil, water breakthroughs faster than its corresponding Houska oil. Therefore, it can be concluded that thixotropy is not so important in influencing the water-flooding operation. As is well-established in the literature [see Ref. 53], in competition with shear-thinning, thixotropy is often realized to be of secondary importance. One can therefore conclude that the prediction that Houska fluids slows down the water breakthrough time is mostly due to its shear-thinning behavior not thixotropic behavior [see, also, Ref. 8].

\section{Figure 7}

For curiosity, we have also investigated the effect of other parameters in the Houska model on the results. Figure 8 shows the effect of the viscosity ratio $(M)$ on the water saturation profiles. From Fig. 8 a it can be concluded that the water breakthrough time decreases with an increase in $\mathrm{M}$. This looks peculiar at first sight, but it must be noted that the effective viscosity of the oil increases by an increase in M (see Fig. $3 b)$. As a result, the mobility of the oil is decreased in comparison with that of water, and so the waterfront should travels faster, as can be seen in Fig 8a. A drop in the mobility of oil should give rise to an increase in the pressure at each cross-section, which is what we see in Fig $8 b$.

\section{Figure 8}

Figure 9 shows the effect of the yield-stress parameter, $\Delta \mathrm{G}$, on the Buckley-Leverett problem. An increase in $\Delta \mathrm{G}$ is seen to increase the pressure gradient needed to mobilize the oil. This lowers the waterphase saturation close to the inlet section, and so the propagation of the waterfront should accelerate, as can be seen in Fig. 9a. The acceleration of the waterfront reduces the breakthrough time and thereby the volume of the recovered oil (see Fig 9b). This means that more oil remains stationary in the reservoir, and so the efficiency of the water flooding operation is decreased this way.

\section{Figure 9}

\subsection{The Five-Spot Problem}

The results found for the one-dimensional Buckley-Leverett problem for waxy crude oils obeying the Houska model are new to our field. To see if similar conclusions can be obtained, at least qualitatively, in more complicated geometries we have decided to carry out similar simulations for the homogenous five- 
spot problem. We had to resort to numerical means for solving the governing equations for the Houska fluid in this challenging benchmark problem (see Appendix C).

Figure 10 shows the time-evolution of the water saturation profile obtained at a typical $\mathrm{Tx}=0.1$. The injected water is seen to mobilize the waxy crude oil (especially near the injection well) and directs it towards the production well. The contours of the water-phase saturation appear to be circular at early stages of the operation. As soon as the breakthrough time is approached, however, the strong pressure gradient in the diagonal direction is predicted to stretch the $S_{w}$ contours so that they become more aligned in the diagonal direction. This notion can better be seen in Fig. 11 which shows the results obtained at $\mathrm{t}=$ 0.2 for $\mathrm{Tx}=0.1$ and $\mathrm{Tx}=100$. This figure shows that by increasing the Tx number, water saturation at the inlet section slightly increases, and this means a longer breakthrough time and a better sweep efficiency-in qualitative agreement with the Buckley-Leverett flow of Houska fluids.

\section{Figure 10}

\section{Figure 11}

Figure 12 shows contours of the structural parameter at two different Tx numbers. This figure shows that, at any given Tx number, the smallest structural parameter occurs near the production well (i.e., at the topright corner). This is not surprising realizing the fact that the shear rate is largest in the vicinity of the production well so that structures are broken down more severely in this area. An increase in the Tx number is seen to significantly alter the structural parameter. This can be attributed to the fact that by an increase in the Tx number, the fluid becomes progressively more shear-thinning. It should be noted that in this figure, regions at which the structural parameter is equal to one are in fact regions which have not yielded. By an increase in the Tx number, more fluid obviously yields but small patches of plug still remain in the domain (see Fig. 12b). This corroborates the experimental finding by Allouche et al. [56] that when a viscoplastic fluid is displaced by a less viscoplastic fluid (say, water), a stagnant layer of the displaced fluid always remains intact near the wall.

\section{Figure 12}

Figure 13 shows the effect of the viscosity ratio, M, on the five spot problem. This figure shows that by an increase in $\mathrm{M}$, water surpasses the waxy oil more easily. This boosts the fractional flow of the water and accelerates the propagation of the waterfront. As a result, the breakthrough time is decreased. On the other hand, a higher viscosity ratio means that more waxy crude remains stationary, and this decreases the sweep efficiency, as can be seen in Fig. 14. This figure shows a comparison between the behavior of a Houska fluid and a Moore fluid having the same viscous behavior. As mentioned earlier for the Buckley- 
Leverett problem, any difference between the two sets of results means the importance of the variable

yield stress in the Houska model. This figure clearly shows that a Houska fluid breakthroughs faster than the Moore model. This means that, like the case for the Buckley-Leverett problem, the variable yield stress of the Houska fluid has a negative effect on the water-flooding operation.

\section{Figure 13}

\section{Figure 14}

Figure 15 shows the importance of the yield stress parameter, $\Delta \mathrm{G}$, in the five-spot problem. This figure clearly shows that, like for the Buckley-Leverett problem, by an increase in $\Delta \mathrm{G}$, the mobility of the oil phase drops. The level of the water-phase saturation also decreases near the injection well when $\Delta \mathrm{G}$ is increased. This means a faster propagation for the water front. That is to say that, the water breakthrough time is decreased by an increase in the yield stress parameter.

\section{Figure 15}

Finally, in Fig. 16 we have compared the behavior of a Bingham fluid with its corresponding Newtonian fluid in terms of the water produced at the production well. To that end, we have switched off timedependent terms by simply setting $T x=0$; we also set $G_{0}=G_{1}$. The Houska model then reuces to the Bingham model. This figure shows by an increase in the yield stress of the oil, more water is produced at the production well. This is as expected because an increase in the yield stress of the oil makes it more difficult for the water to displace it.

\section{Figure 16}

\section{Concluding Remarks}

Based on the results obtained in the present work, it is concluded that the non-Newtonian behavior of waxy oils obeying the Houska model has a decisive role in the water-flooding operation. An increase in the breakdown-to-rebuild ratio in this rheological model is predicted to increase the time needed for the water to breakthrough; the sweep efficiency is also increased when this ratio is increased. The effect was attributed to the shear-thinning behavior of the Houska fluid which eclipses its thixotropic behavior when this ratio is large. And, when this ratio is so small that shear-thinning becomes less important, no significant difference could be found between the response of a Houska model with that of a Bingham model. This suggests that in competition with shear-thinning, a waxy oil's thixotropic behavior is of secondary importance in water-flooding operation. An increase in the viscosity ratio and/or the yield stress ratio in the Houska model is also predicted to aggravate the water breakthrough phenomenon. To 
focus on the role played by the fluid's variable yield stress, we have relied on a Moore model which has the same viscous behavior as the Houska model. The difference between the two sets of results revealed that the variable yield stress in the Houska model has an accelerating effect on the water flooding operation. Our numerical results obtained in the five spot problem are realized to be in qualitative agreement with those found in the Buckley-Leverett problem suggesting that the latter (simpler) flow might be adequate for fundamental studies dealing with waxy oils in ensuing works. As to the significance of the results obtained in this work, it must be stressed that they are new to the field of liquid/liquid displacement. Still, we would like to stress that describing the rheology of a complex fluid in terms of a locally-averaged effective viscosity together with the use of a mean flow velocity-instead of the local pore velocity-seems rather coarse and awaits experimental and/or theoretical verification. Another important issue which can undermine the validity of this law is the recent finding by Allouche et al [56] who have shown that when a viscoplastic fluid is displaced by a less viscoplastic fluid (say, water) a stagnant layer of the fluid remains intact near the wall. The validity of the Darcy's law under such a condition is still an open question to be addressed in subsequent works. Until such issues are completely resolved, we feel entitled to employ the (coarse) Darcy's model in order to gain a better insight as to the mechanisms involved in the water-flooding operation of waxy crude oils. The results reported in this work can also serve as a baseline with which the performance of more accurate pore-scale and/or network models can be evaluated in future works.

\section{Acknowledgement}

The authors wish to express their sincerest thanks to Iran National Science Foundation (INSF) for supporting this work under contract number 95815139. Special thanks are also due to the respectful reviewers for their constructive comments.

\section{List of Symbols:}

$\begin{array}{ll}\text { a: } & \text { Structural re-build parameter } \\ \mathrm{b}: & \text { Structural destruction parameter } \\ \mathrm{G}_{0}: & \text { Infinite-shear threshold pressure gradient } \\ \mathrm{G}_{1}: & \text { Zero-shear threshold pressure gradient } \\ \mathrm{k}: & \text { Reservoir's permeability } \\ \mathrm{k}_{\mathrm{r}}: & \text { Relative permeability } \\ \mathrm{M}: & \text { Viscosity ratio } \\ \mathrm{N} & \text { Yield stress ratio } \\ \mathrm{p}: & \text { Pressure }\end{array}$




$\begin{array}{ll}\mathrm{q}: & \text { Rate of injection/production } \\ \mathrm{Q}_{\mathrm{in}, \mathrm{w}}: & \text { Volumetric flow rate of water injection } \\ \mathrm{R}: & \text { Capillary tube radius } \\ \mathrm{S}: & \text { Saturation } \\ \mathrm{t}: & \text { Time } \\ \mathrm{Tx}: & \text { Thixotropy number } \\ \mathbf{u}: & \text { Velocity vector } \\ \mathrm{u}: & \text { Velocity component in } \mathrm{x} \text { direction } \\ \mathrm{v}: & \text { Velocity component in y direction } \\ \phi: & \text { Porosity } \\ \dot{\gamma}: & \text { Effective shear rate } \\ \eta_{0}: & \text { Infinite-shear viscosity } \\ \eta_{1}: & \text { Zero-shear viscosity } \\ \lambda: & \text { Structural parameter } \\ \mu: & \text { Dynamic viscosity } \\ \rho: & \text { Density } \\ \tau_{\mathrm{y} 0}: & \text { Infinite-shear yield stress } \\ \tau_{\mathrm{y} 1}: & \text { Zero-shear yield stress } \\ & \end{array}$

\section{Subscripts:}

$\begin{array}{ll}\text { o } & \text { Oil } \\ \text { w } & \text { Water } \\ \mathrm{r} & \text { Relative }\end{array}$




\section{References:}

[1] G.P. Willhite, Water Flooding, Society of Petroleum Engineers, 3 (1986).

[2] T. Sochi, Non-Newtonian flow in porous media, Polymer, 51 (2010) 5007-5023.

[3] K. Aziz, A. Settari, Petroleum reservoir simulation, Chapman \& Hall (1979).

[4] Z. Chen, G. Huan, Y. Ma, Computational methods for multiphase flows in porous media, Siam (2006).

[5] N. Ezekwe, Petroleum reservoir engineering practice, Pearson Education (2010).

[6] T. A1-Fariss, K.L. Pinder, Flow of a shear-thinning liquid with yield stress through porous media, SPE 14683 (1985).

[7] Y-S. Wu, K. Pruess, P.A. Witherspoon, Flow and displacement of Bingham non- Newtonian fluids in porous media, SPE Reservoir Engineering, (1992) 369-76.

[8] X. Yi, Model for displacement of Herschel-Bulkley non-Newtonian fluid in porous media and its application in fracturing fluid cleanup, SPE 86491 (2004).

[9] T. Chevalier, C. Chevalier, X. Clain, J.C. Dupla, J. Canou, S. Rodts, P. Coussot, Darcy's law for yield stress fluid flowing through a porous medium, J. of Non-Newtonian Fluid Mechanics, 195 (2013) 57-66.

[10] T. Chevalier, L. Talon, Generalization of Darcy's law for Bingham fluids in porous media: From flow-field statistics to the flow-rate, Physical Review E 91, (2015) 23011-6.

[11] L.T. Wardhaugh, D.V. Boger, Flow characteristics of waxy crude oils: application to pipeline design, AIChE Journal, 37(6) (1991) 871-885.

[12] C.J. Dimitriou, G.H. McKinley, R. Venkatesan, Rheo-PIV analysis of the yielding and flow of model waxy crude oil, Energy and Fuels, 25 (2011) 3040-3052.

[13] J.R.A. Pearson, P.M.J. Tardy, Models for flow of non-Newtonian and complex fluids through porous media, J. Non-Newtonian Fluid Mech., 102 (2002) 447-473.

[14] D. Pritchard, J.R.A. Pearson, Viscous fingering of a thixotropic fluid in a porous medium or a narrow fracture, J. Non-Newtonian Fluid Mech., 135 (2006) 117-127.

[15] F. Bautista, J.M. de Santos, J.E. Puig, O. Manero, Understanding thixotropic and antithixotropic behavior of viscoelastic micellar solutions and liquid crystalline dispersions, J. non-Newtonian Fluid Mech, 80 (1999) 93-113.

[16] M. Houska, Engineering Aspects in the Rheology of Thixotropic Fluids, PhD Thesis, CVUT Prague (1981).

[17] S.E. Buckley, M.C. Leverett, Mechanism of fluid displacement in sands, Petroleum Technology, (1941) 107-116.

[18] B.H. Caudle, M.D. Witte, Production potential changes during sweep-out in a five-spot system, Trans. AIME, 216 (1959) 446.

[19] T. Ertekin, J.H. Abou-Kassem, G.R. King, Basic applied reservoir simulation, SPE Text Book 
Series (2001)

[20] Z. Chen, Reservoir Simulation: Mathematical Techniques in Oil Recovery, Siam (2007).

[21] J.J. Zhang, X. Liu, Some advances in crude oil rheology and its application, J. Cent. South Univ. Technol., 15(s1) (2008) 288-292.

[22] H.P. Ronningsen, Rheological behaviour of gelled, waxy North Sea crude oils, J. Petroleum Sci. Eng., 7 (1992) 177-213.

[23] M. Kane, M. Djabourov, J.L. Volle, Rheology and structure of waxy crude oils in quiescent and under shearing conditions, Fuel, 83 (2004) 1591-1605.

[24] R.F.G. Visintin, R. Lapasin, E. Vignati, P. D'Antona, T.P. Lockhart, Rheological behavior and structural interpretation of waxy crude oil gels, Langmuir, 21 (2005) 6240- 6249.

[25] R.F.G. Visintin, T.P. Lockhart, R. Lapasin, P. D’Antona, Structure of waxy crude oil emulsion gels, J. Non-Newtonian Fluid Mech., 149 (2008) 34-39.

[26] O. Adeyanju, L. Oyekunle, An experimental study of rheological properties of Nigerian waxy crude oil, Petroleum Science and Technology, 30 (2012) 1102-1112.

[27] E.A. Kirsanov, S.V. Remizov, Application of the Casson model to thixotropic waxy crude oil, Rheol Acta, 38 (1999) 172-176.

[28] A. Fasano, L. Fusi, S. Correra, Mathematical models for waxy crude oils, Meccanica, 39 (2004) 441-482.

[29] E. Ghanaei, D. Mowla, D. Prediction of waxy oil rheology by a new model-ACS Publications, Energy Fuels, 24 (2010) 1762-1770.

[30] C.J. Dimitriou, G.H. McKinley, A comprehensive constitutive law for waxy crude oil: a thixotropic yield stress fluid, Soft Matter, 10 (2014) 6619-6644.

[31] P.R. de Souza Mendes, Modeling the thixotropic behavior of structured fluids, J. Non-Newton. Fluid Mech., 164 (2009) 66-75.

[32] K. Dullaert, J. Mewis, A structural kinetics model for thixotropy, J. Non-Newtonian Fluid Mech. 139 (2006) 21-30.

[33] X.D. Zhao, Study on the unsteady hydraulic and thermal computation of the restart process of the PPD-beneficiated crude oil pipeline, M.Sc. thesis, Beijing: China University of Petroleum (1999).

[34] R.G. Larson, Constitutive equations for thixotropic fluids, Journal of Rheology, 59 (2015) 595611.

[35] L. Guo, J. Zhang, S. Han, H. Teng, Evaluation of thixotropic models for waxy crudes based on stepwise shearing measurements, Petroleum Science and Technology, 31 (2013) 895-901.

[36] J. Sestak, M.E. Charles, M.G. Cawkwell, M. Houska, Start-up of gelled crude oil pipelines, J. Pipelines, 6 (1987) 15-24.

[37] G. Vinay, and A. Wachs, A 1.5D Numerical model for weakly compressible viscoplastic and thixotropic flows: application to the start-up of waxy crude oils in pipeline, The $\mathrm{XV}^{\text {th }}$ 
International Congress on Rheology, The Society of Rheology $80^{\text {th }}$ Annual Meeting, edited by A. Co, L.G. Leal, R.H. Colby, and A.J. Giacomin.

[38] A. Wachs, G. Vinay, I. Frigaard, A 1.5D numerical model for the start-up of weaklycompressible flow of a viscoplastic and thixotropic fluid in pipelines, Journal of Non-Newtonian Fluid Mechanics, 159 (2009) 81-94.

[39] A. Ahmadpour, K. Sadeghy, S.R. Maddah-Sadatieh, The effect of a variable plastic viscosity on the restart problem of pipelines filled with gelled waxy crude oils, J. Non-Newtonian Fluid Mech., 205 (2014) 16-27.

[40] H. Teng, J. Zhang, A new thixotropic model for waxy crude, Rheol Acta, 52 (2013) 903-911.

[41] R. Mendes, G. Vinay, G. Ovarlez, P. Coussot, Modeling the rheological behavior of waxy crude oils as a function of flow and temperature history, J. Rheol., 59(3) (2015) 703-732.

[42] J. J. Derksen, Drag on random assemblies of spheres in shear-thinning and thixotropic liquids, Physics of Fluids, 21 (2009) 083302-9.

[43] R.P. Chhabra, Bubbles, Drops, and Particles in Non-Newtonian Fluids, CRC Press, Boca Raton FL, Vol. 2, 1993.

[44] R.M. McKinley, H.O. Jahns, W.W. Harris, R.A. Greenkorn, Non-newtonian flow in porous media, 12(1) (1966) AIChE J, 17-20.

[45] D. Yang, R.P. Currier, D.Z. Zhang, Ensemble phase averaged equations for multiphase flows in porous media. Part 1: The bundle-of-tubes model, Int. J. Multiphase Flow, 35 (2009) 628639.

[46] A. Ahmadpour, K. Sadeghy, An exact solution for laminar, unidirectional flow of Houska thixotropic fluids in a circular pipe, Journal of Non-Newtonian Fluid Mechanics, 194 (2013) 2331.

[47] R.B. Bird, R.C. Armstrong, O. Hassager, Dynamics of Polymeric Liquids, John Wiley \& Sons (1987).

[48] A. Salehi-Shabestari, Simulating Flow and Displacement of Non-Newtonian Fluids in Homogeneous Porous Media, $\mathrm{PhD}$ thesis, in progress.

[49] Y-S. Wu, and K. Pruess, A numerical method for simulating non-Newtonian fluid flow and displacement in porous media, Advances in Water Resources, 21 (1998) 351-362.

[50] H.J. Welge, A simplified method for computing oil recovery by gas or water drive, Journal of Petroleum Technology, 4 (1952) 91-98.

[51] F. Moore, The rheology of ceramic slips and bodies, Trans. Br. Ceram. Soc., 58 (1959) 470-494.

[52] D.A. Weiss, A. Reinl , D.M. Binding, Spray impact on solid walls of non-Newtonian fluids, including yield stress and thixotropic behavior, XXI ICTAM proceedings, 15-21 August 2004, Warsaw, Poland.

[53] M.P. Escudier, I.W. Gouldson, D.M. Jones, Taylor vortices in Newtonian and shearthinning liquids, Proc. R. Soc. Lond. Math. phys. Sci., 449 (1995)155-176. 
[54] H.B. Crichlow, Modern Reservoir Engineering-A Simulation Approach. Englewood Cliffs, NJ: Prentice Hall, 1977.

[55] Z. Chen, G. Huan, B. Li, An improved IMPES method for two-phase flow in porous media, Transport in Porous Media, 54(3) (2004) 361-376.

[56] M. Allouche, I.A. Frigaard, and G. Sona, Static wall layers in the displacement of two viscoplastic fluids in a plane channel, J. Fluid. Mech., 224 (2000) 243-277.

\section{List of Figures:}

Figure 1: Frontal advance of the injected water in the one-dimensional Buckley-Leverett problem.

Figure 2: The five-spot problem used for simulating the water flooding operation (circles refer to the production wells with triangles referring to the injection wells).

Figure 3: Effect of different parameters in the Houska model on its effective viscosity in simple shear: (a) effect of thixotrpy number, Tx, obtained at $\mathrm{G}_{0}=0.2, \mathrm{G}_{1}=0.25, \mathrm{M}=0.8$, (b) effect of the viscosity ratio, $M$, obtained at $\mathrm{G}_{0}=0.2, \mathrm{G}_{1}=0.25, \mathrm{Tx}=0.1$, and (c) effect of the yield stress parameter, $\Delta \mathrm{G}$, obtained at $\mathrm{Tx}=0.1$, and $\mathrm{M}=0.8$.

Figure 4: Effect of the thixotropic parameter, Tx, on: (a) the water saturation profile, (b) pressure variation, and (c) volume of the recovered oil for a Houska fluid $\left(\mathrm{M}=0.8, \mathrm{G}_{0}=0.2, \mathrm{G}_{1}=0.25\right.$, and $\mathrm{t}=$ $0.02)$.

Figure 5: Effect of the thixotropic number, Tx, on: (a) the water saturation profile, (b) volume of the recovered oil obtained for a Moore fluid $\left(M=0.8, G_{0}=0.2, G_{1}=0.25\right.$, and $\left.t=0.02\right)$.

Figure 6: A comparison between Moore and Houska model predictions: (a) the water saturation profile, (b) recovered oil obtained at $\mathrm{Tx}=100\left(\mathrm{M}=0.8, \mathrm{G}_{0}=0.2, \mathrm{G}_{1}=0.25, \mathrm{t}=0.02\right)$.

Figure 7: Effect of the thixotropic number, Tx, on the water saturation profile obtained at $\mathrm{M}=1$, $\mathrm{G}_{0}=0.2, \mathrm{G}_{1}=0.25$, and $\mathrm{t}=0.02$.

Figure 8: Effect of the viscosity ratio, $M$, on: (a) water saturation profile, and (b) pressure variation (obtained at $\mathrm{Tx}=0.1, \mathrm{G}_{0}=0.2, \mathrm{G}_{1}=0.25$, and $\mathrm{t}=0.02$ ).

Figure 9: Effect of the yield stress parameter, $\Delta \mathrm{G}$, on: (a) water saturation profile, (b) pressure profile at, $\mathrm{Tx}=0.1, \mathrm{M}=0.8$, and $\mathrm{t}=0.02$.

Figure 10: Time evolution of the water saturation contours in the five-spot problem of Houska fluids obtained at: (a) $\mathrm{t}=0.005$, (b) $\mathrm{t}=0.01$, (c) $\mathrm{t}=0.015$, (d) $\mathrm{t}=0.02\left(\mathrm{G}_{0}=0.2, \mathrm{G}_{1}=0.25, \mathrm{Tx}=0.1\right.$, and $\mathrm{M}=$ $0.8)$.

Figure 11: Effect of the Tx number in the Houska model on the water saturation contours in the five-spot problem: (a) $\mathrm{Tx}=0.01$, (b) $\mathrm{Tx}=100\left(\mathrm{G}_{0}=0.2, \mathrm{G}_{1}=0.25, \mathrm{Tx}=0.1\right.$, and $\left.\mathrm{M}=0.8\right)$.

Figure 12: Contours of the structural parameter, $\lambda$, obtained at: (a) $\mathrm{Tx}=0.1$, (b) $\mathrm{Tx}=1 .\left(\mathrm{G}_{0}=0.2, \mathrm{G}_{1}=\right.$ $0.25, \mathrm{M}=0.8)$. 
Figure 13: Effect of the viscosity ratio, $M$, on water saturation contours in the five-spot problem: (a) $\mathrm{M}=$ 0.2 , (b) $\mathrm{M}=1\left(\mathrm{G}_{0}=0.2, \mathrm{G}_{1}=0.25, \mathrm{Tx}=0.1\right.$, and $\left.\mathrm{t}=0.02\right)$.

Figure 14: A comparison between water saturation profiles for Moore model (left plot) and Houska model (right plot) obtained at $\mathrm{G}_{0}=0.2, \mathrm{G}_{1}=0.25(\mathrm{M}=0.8, \mathrm{t}=0.02, \mathrm{Tx}=100)$.

Figure 15: Effect of $\Delta \mathrm{G}$ in the Houska model on water saturation contours in the five-spot problem:

(a) $\Delta \mathrm{G}=0.1$ (b) $\Delta \mathrm{G}=0.3(\mathrm{Tx}=0.1, \mathrm{M}=0.8$, and $\mathrm{t}=0.02)$.

Figure 16: Comparison between Bingham and Newtonian fluid in terms of the volume of water produced at the production well.

Figure A1: Variation of the waxy crude oil's apparent viscosity with temperature at $\dot{\gamma}=50 \mathrm{~s}^{-1}$.

Figure A2: The hysteresis loop for a waxy crude oil obtained at $\mathrm{T}=15^{\circ} \mathrm{C}$.

Figure A3: The flow-curve obtained at different resting periods (RP) for a commercial waxy crude oil obtained at $\mathrm{T}=15^{\circ} \mathrm{C}$.

Figure A4: Performance of the Houska model for fitting unsteady shear data for a commercial waxy crude oil obtained at $\mathrm{T}=1{ }^{\circ} \mathrm{C}$. : (a) stepwise increase in shear rate, (b) the measured shear stress.

Figure B1: Effect of the decay time, $\Lambda$, on the time-evolution of the structural parameter obtained in simple shear for: $\lambda_{\text {eq }}=0 ; \dot{\gamma}=1$.

Figure C1: Computational domain used for simulating the five-spot problem.

Figure C2: A comparison between our numerical results (symbols) and our theoretical results (lines) obtained for the Houska fluid $\left(\mathrm{G}_{0}=0, \mathrm{G}_{1}=0.12, \mathrm{M}=0.2\right)$. 


\section{Appendix A: Rheology of a Commercial Waxy Crude Oil from the Middle East}

To characterize the rheological behavior of a commercial waxy crude oil extracted from a reservoir in the Middle East, a variety of rheological data were obtained using the Anton-Paar MCR 102 rheometer in the concentric-cylinder mode. The first set of experiments were conducted in order to determine the oil's "wax appearance temperature" (WAT). To achieve this goal, the sample was heated in order to dissolve any precipitated wax already present in the sample. The temperature was then lowered continuously with a rate of $1{ }^{\circ} \mathrm{C} / \mathrm{min}$ during which the sample's viscosity was measured at the constant shear rate of $50 \mathrm{~s}^{-1}$. Figure A1 shows the apparent viscosity of the sample as a function of the temperature. As can be seen in this figure, at temperatures lower than roughly $16{ }^{\circ} \mathrm{C}$, the viscosity of the oil starts to increase sharply inferring that the wax appearance temperature of the sample has been reached. (Interestingly, this figure shows that the Arrhenius equation is a good fit for temperatures higher than $16{ }^{\circ} \mathrm{C}$.)

\section{Figure A1}

The rheological behavior of waxy crude oils have been reported to deviate dramatically from the Newtonian behavior even at temperatures slightly lower than WAT. Figures A2 and A3 show that this is indeed the case for our waxy crude oil at $15{ }^{\circ} \mathrm{C}$ (which is just slightly lower than the WAT for this oil). In fact, based on the hysteresis results presented in Fig. A2 one can conclude that this oil is definitely thixotropic. On the other hand, from the rheograms presented in Fig. A3 it can be concluded that the sample is viscoplastic and exhibits a yield stress which is time-dependent.

\section{Figure A2}

\section{Figure A3}

To see if the Houska model can indeed represent this waxy oil, the model prediction had to be compared with the data gathered from certain rheological tests. To that end, we have decided to rely on the data obtained from the "step shear rate" test. Figure A4a shows such a test conducted at $1{ }^{\circ} \mathrm{C}$ on our oil sample. And, in Fig. A4b we have shown the measured shear stress together with the Houska's model fit. As can be seen in Fig. A4b, Houska's model is doing a nice job in fitting the data for this oil (particularly at longer times) which means that it can safely be used for representing the rheology of our waxy crude oil.

\section{Figure A4}

Based on the results presented above, we have: $a=0.007 \mathrm{~s}^{-1}, \mathrm{~b}=0.047$. Therefore, the breakdown-torebuild ratio for this oil is roughly equal to $b / a \approx 7$. Similarly, the two viscosity coefficients for this oil are determined as: $\eta_{0}=0.086 \mathrm{~Pa}, \eta_{1}=0.108 \mathrm{~Pa}$ so that the viscosity ratio for this oil is roughly equal to $M=\eta_{0} / \eta_{1}=0.8$. In addition, the two yield stresses of the fluid are obtained as: 
$\tau_{\mathrm{y}, 0}=0.155 \mathrm{~Pa}, \tau_{\mathrm{y}, 1}=0.182 \mathrm{~Pa}$ which means that the yield stress ratio for this oil is roughly equal to $\mathrm{N}=\tau_{\mathrm{y}, 0} / \tau_{\mathrm{y}, 1}=0.85$. These values can give us an idea as to the size of these parameters at the time of the numerical simulation (see Section 3). Still, we would like to stress that we do not believe that this crude oil would represent all possible waxy crude oils in a global sense. They can obviously have different M, $\mathrm{N}$, and b/a ratios while still obeying the Houska model. 


\section{Appendix B: Importance of the Destruction-to-Rebuild Ratio (b/a)}

As mentioned in the main text, the destruction-to-rebuild ratio, b/a, controls the degree of Houska fluid's shear-thinning behavior. In fact, the shear-thinning effect becomes more pronounced when this ratio is increased. It can be shown that this ratio also controls the degree of fluid's thixotropy. To show this in better perspective, let's assume that the fluid lacks any yield stress. The Houska fluid's thixotropy then reflects itself in just the fluid's viscosity which is allowed to be time-dependent. In viscometric flows, the apparent viscosity of a Houska model can be written as:

$\eta(t, \dot{\gamma})=\eta_{\infty}+\left(\eta_{0}-\eta_{\infty}\right) \lambda$

As mentioned earlier, the kinetic equation is:

$\mathrm{D} \lambda / \mathrm{Dt}=\mathrm{a}(1-\lambda)-\mathrm{b} \lambda \dot{\gamma}$

If we integrate the kinetic equation from the initial value of $\lambda=1$ to its equilibrium value of $\lambda_{\text {eq }}$, we obtain:

$\lambda(\mathrm{t})=\lambda_{\text {eq }}-\left(\lambda_{\text {eq }}-1\right) \exp (-t / \Lambda)$.

The structural decay is seen to be controlled by $\Lambda$ which is:

$$
\Lambda=\frac{1}{\mathrm{a}+\mathrm{b} \dot{\gamma}}
$$

This parameter has the dimension of time, and so it can be called the decay time. Equation B4 shows that the decay time plays the same role for thixotropic fluids as the relaxation time plays for elastic fluids. That is, fluids having larger $\Lambda$ are expected to exhibit thixotropic effects more profoundly because they need longer times to attain their equilibrium viscosity. The decay time can be made dimensionless when multiplied by the rebuild parameter, a, so that we have:

$$
\Lambda^{*}=\mathrm{a} \Lambda=\frac{1}{1+(\mathrm{b} / \mathrm{a}) \dot{\gamma}} \text {. }
$$

Obviously, in simple shear, the dimensionless decay time depends only on the b/a ratio. Figure B1 shows a plot of the structural parameter versus time for different values of the dimensionless decay time in simple shear; for convenience, the asterisk has been removed from $\Lambda^{*}$. This figure shows that for fluid's thixotropy to be important, the decay time must be large (or, equivalently, the b/a ratio must be small). That is to say that, by working at $b / a>>1$ we are practically capturing the shear-thinning aspect of the fluid whereas by working at $b / a<<1$ we are mainly focusing on the thixotropic aspect of the fluid. At intermediate values of $b / a$ both effects are operational with varying degrees.

\section{Figure B1}




\section{Appendix C: Numerical Method}

We had to resort to numerical means for solving the equations governing the five-spot problem. To that end, the governing equations were descritized through the finite difference method on a uniform square block-centered $25 \times 25$ mesh. This mesh size was realized to ensure grid-independent results [48]. To discretize the second-order spatial derivatives in the left-hand side of Eqs. 4 and 5, we have relied on the central-difference scheme. For the temporal term, the backward differencing scheme is used. To linearize the discretized equations use was made of the explicit scheme in which the relative permeability and viscosity are calculated from the pressure update. To solve the linearized equations, we have employed the implicit pressure/explicit saturation (IMPES) method [54,55]. The main idea behind IMPES method is to solve the pressure equation implicitly while the saturation equation is updated explicitly. This greatly lowers the computational error thanks to the number of equations being reduced by half (as compared to the fully-implicit method). To implement this method, the water phase saturation can be dropped from the flow equations by first adding up Eqs. 4 and 5 together; the pressure field is then solved implicitly. Knowing the pressure field, the water phase saturation is calculated explicitly by solving the water phase equation. Using explicit linearization and IMPES method of solution lowers the amount of computational work required for each time step significantly. This is because there is no need for any iterations to solve the equations. On the other hand, this method limits our choice of the time-step size to quite small values in order to ensure numerical stability. In practice, this numerical scheme turned out to be well capable of producing converged results within a reasonable CPU time for our fluid mechanics problem. A Fortran code was developed to carry out these computations. To verify the code, it was used to reproduce the classic Newtonian/Newtonian displacement results for the one-dimensional Buckley-Leverett problem [17]. As a more stringent test, the code was used to reproduce the graphical (exact) results obtained in this work for the Houska fluid in the Buckley-Leverett problem (see Section 3.1). Figure C1 shows the flow domain used for the simulation. Thanks to its symmetry, only a quarter of the original five-well configuration has been used as the computational domain. To proceed with the simulations, the same properties as used for the Buckley-Leverett problem have been adopted for the five-spot problem, too. As can be seen in Fig. $\mathrm{C} 1$ the code is doing a nice job for the Houska fluids in the Buckley-Leverett problem.

\section{Figure C1}

Figure C2 


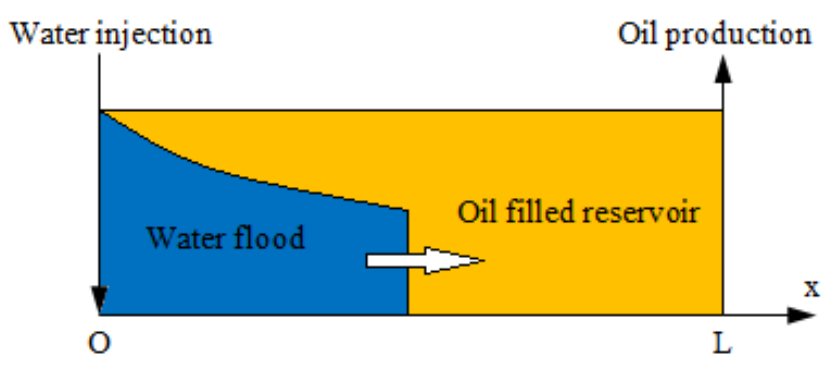

Figure 1

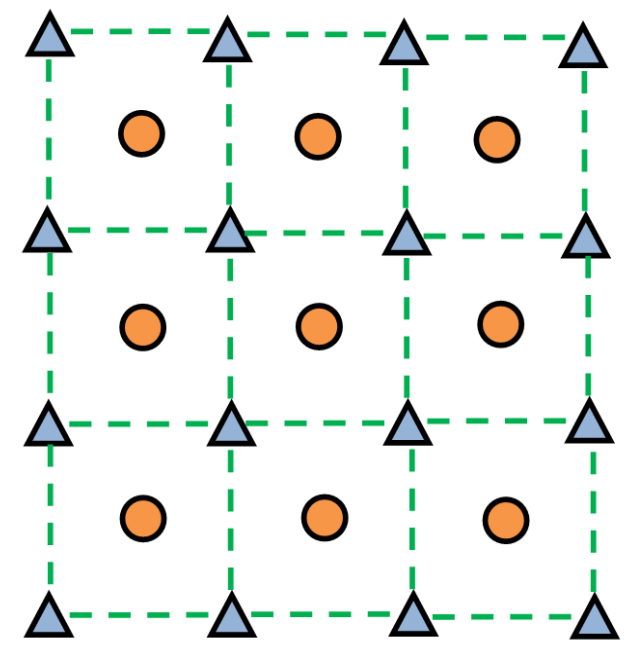

Figure 2 


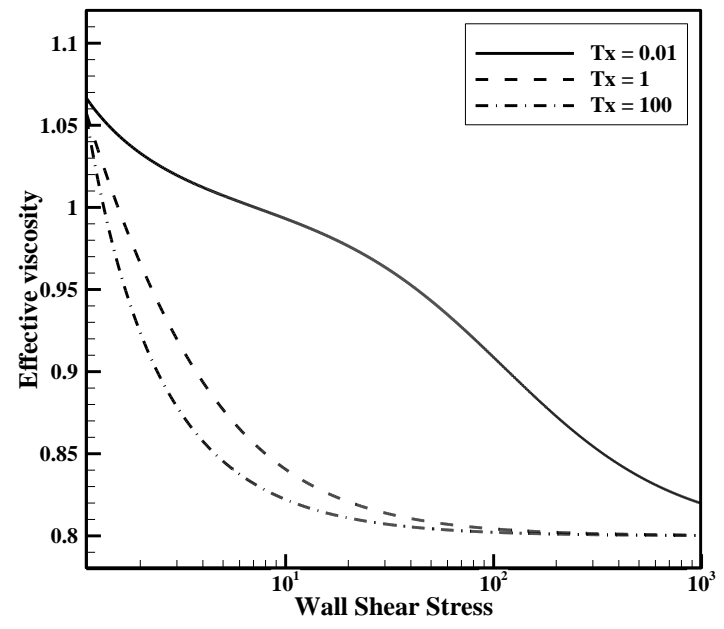

(a)

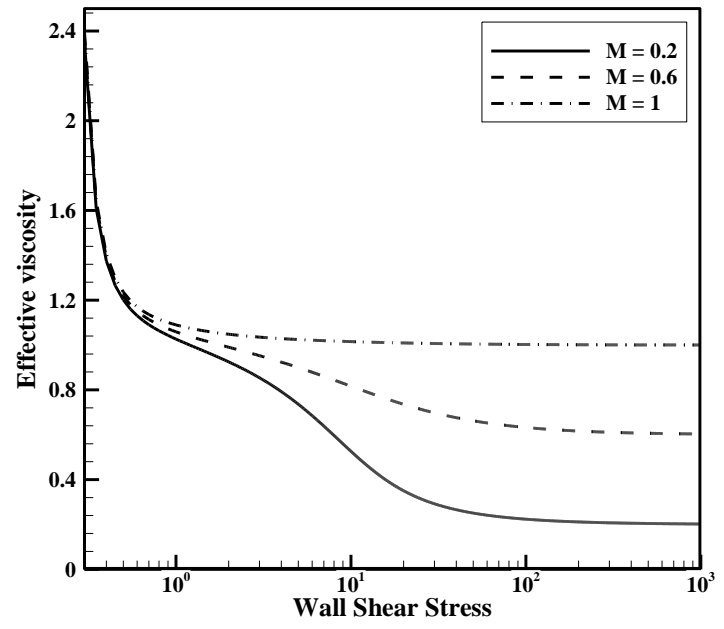

(b)

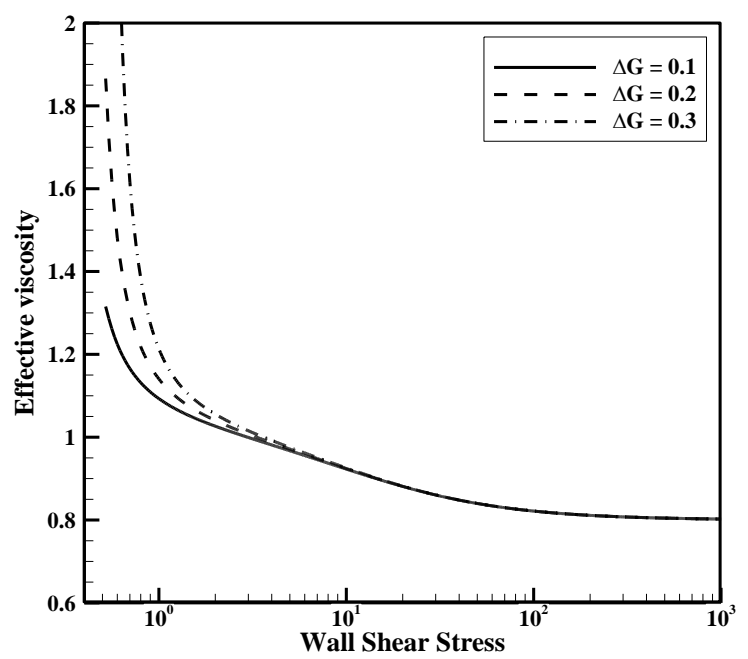

(c)

Figure 3 


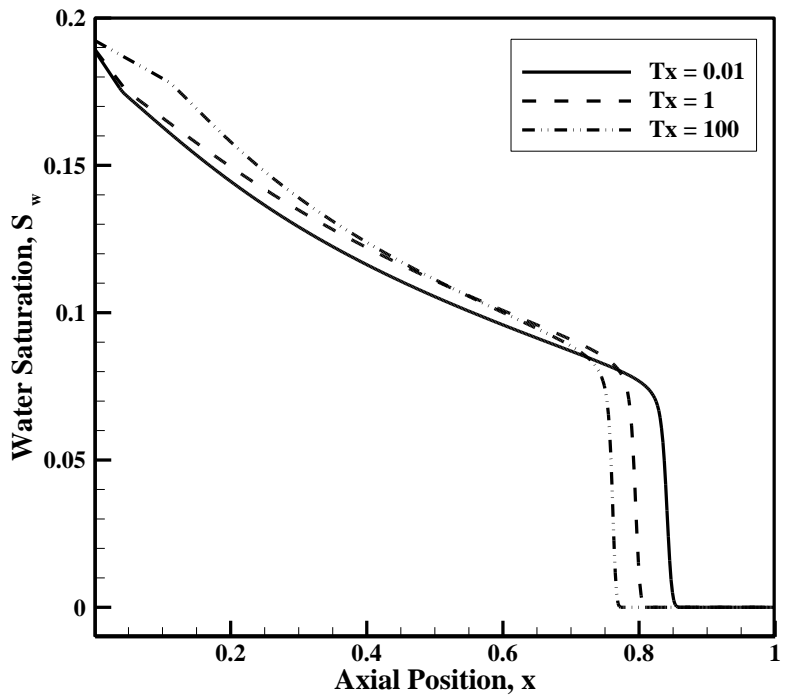

(a)

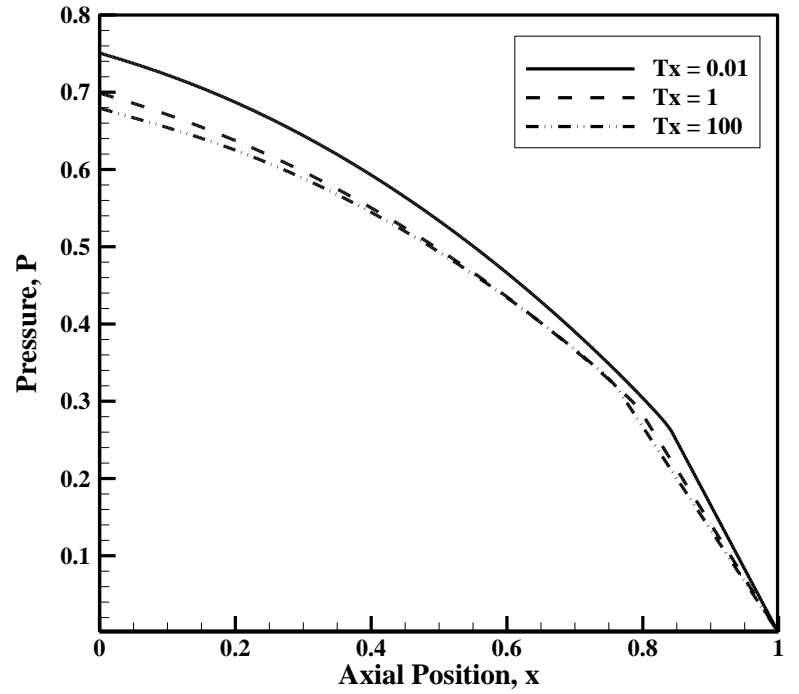

(b)

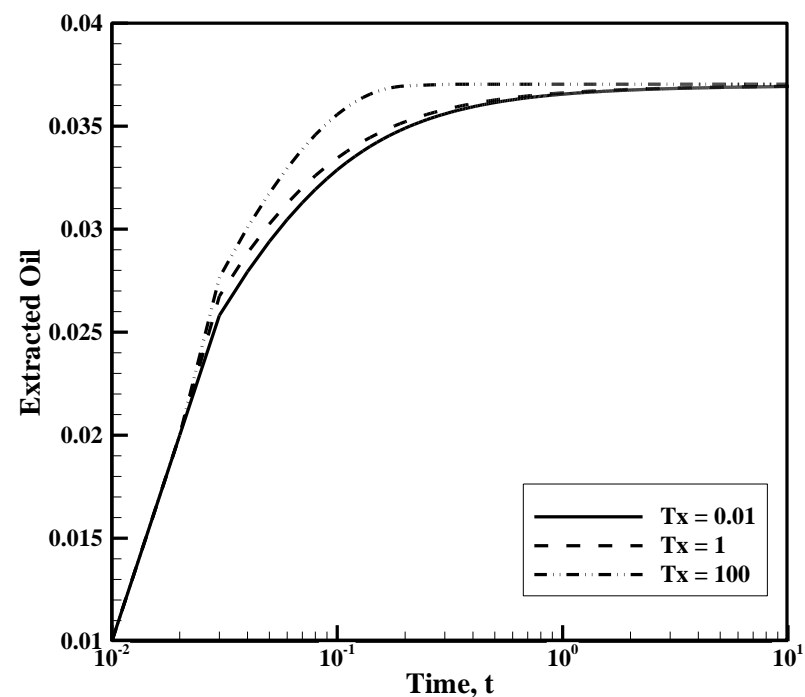

(c)

Figure 4 


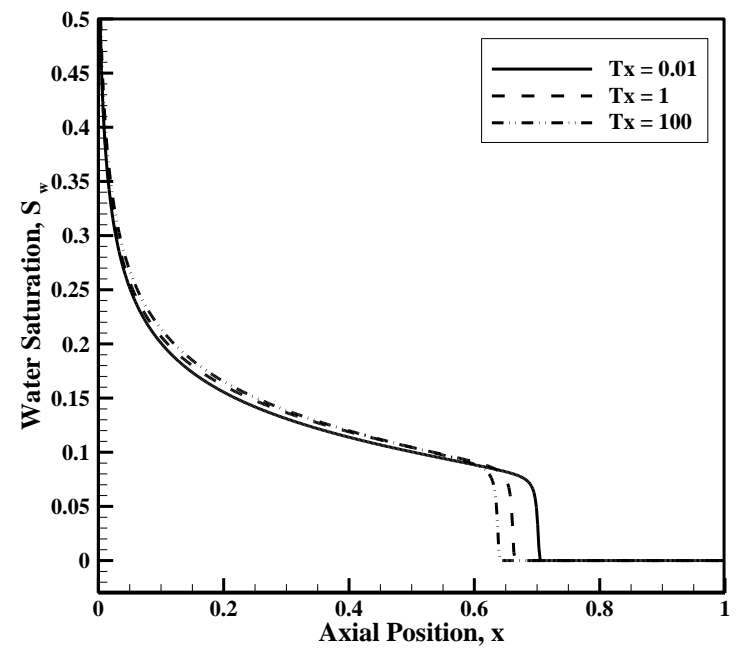

(a)

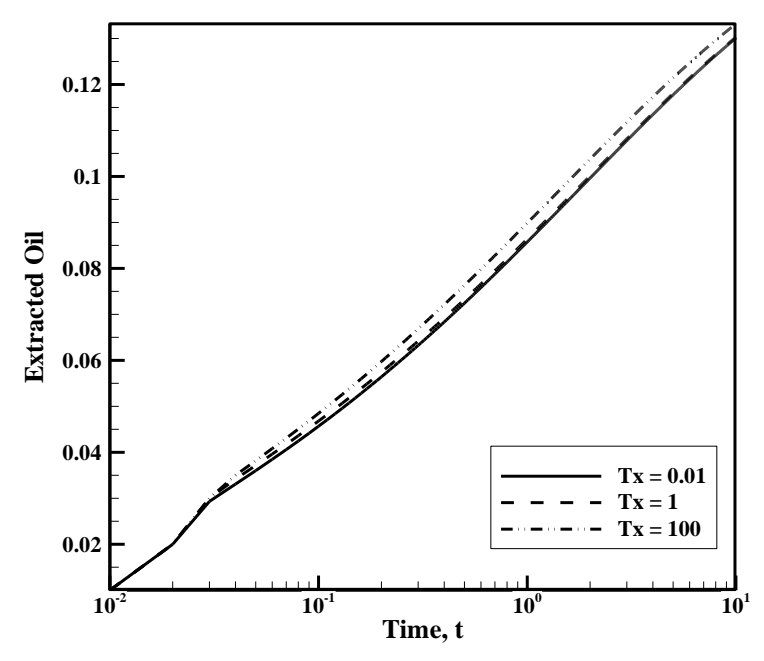

(b)

Figure 5

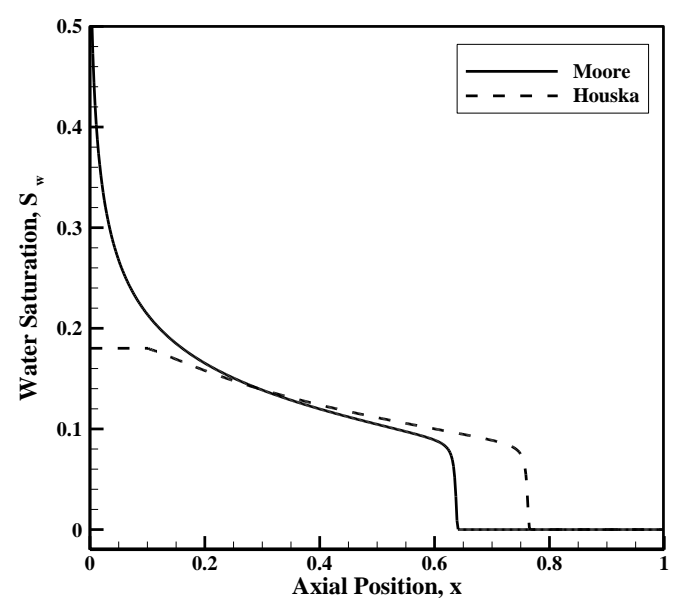

(a)

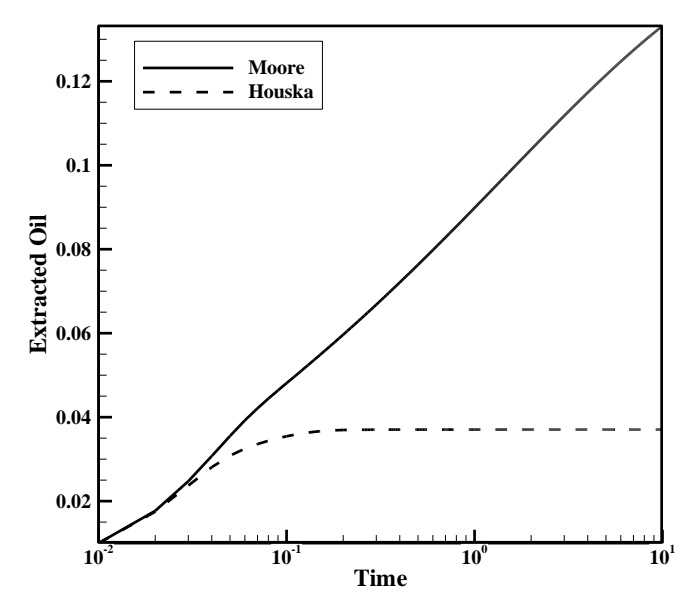

(b)

Figure 6 


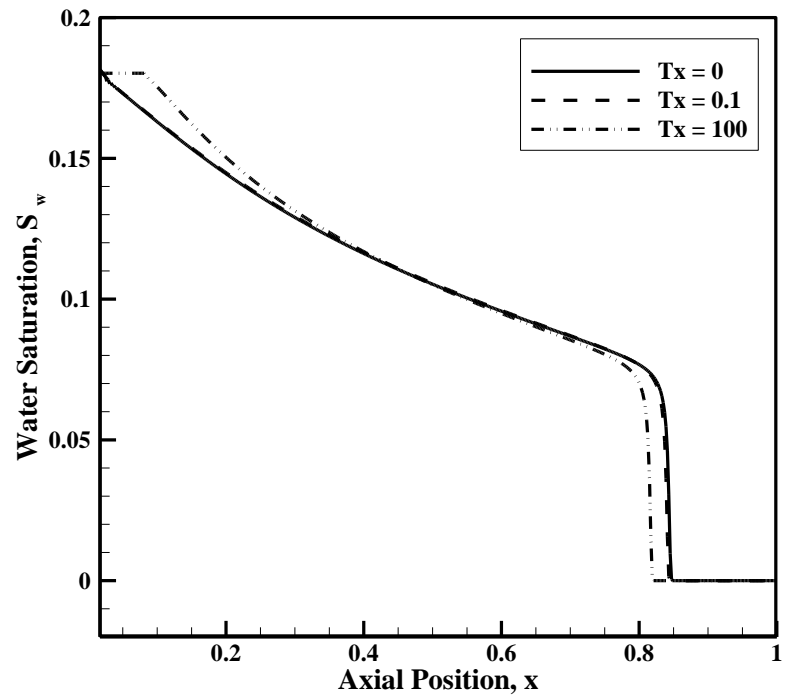

Figure 7

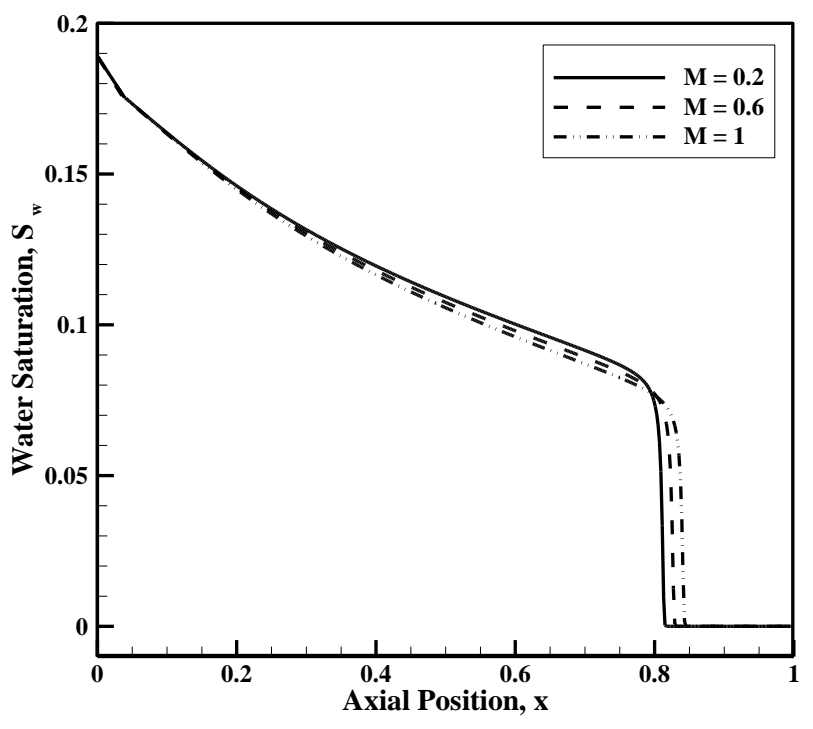

(a)

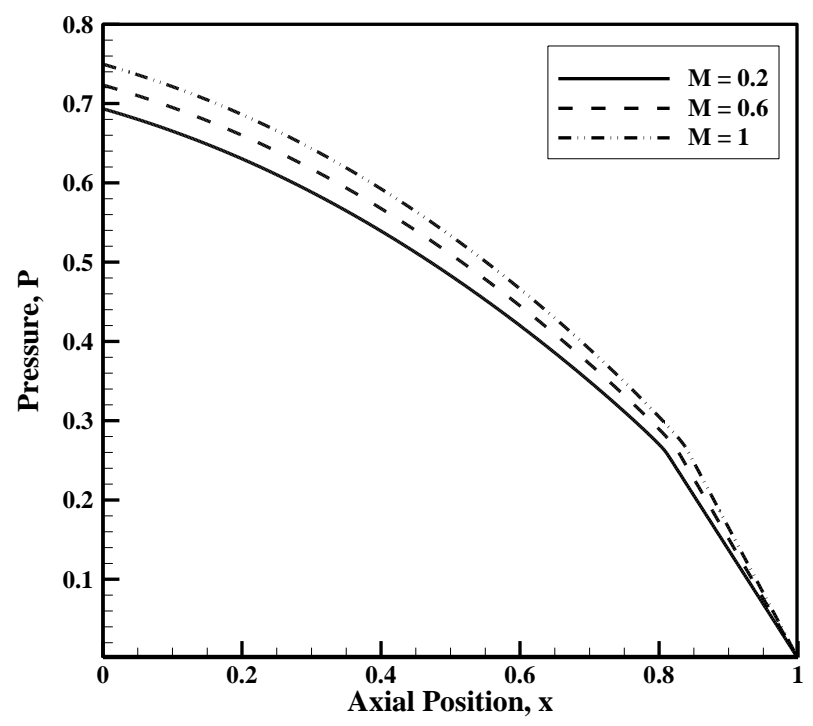

(b)

Figure 8 


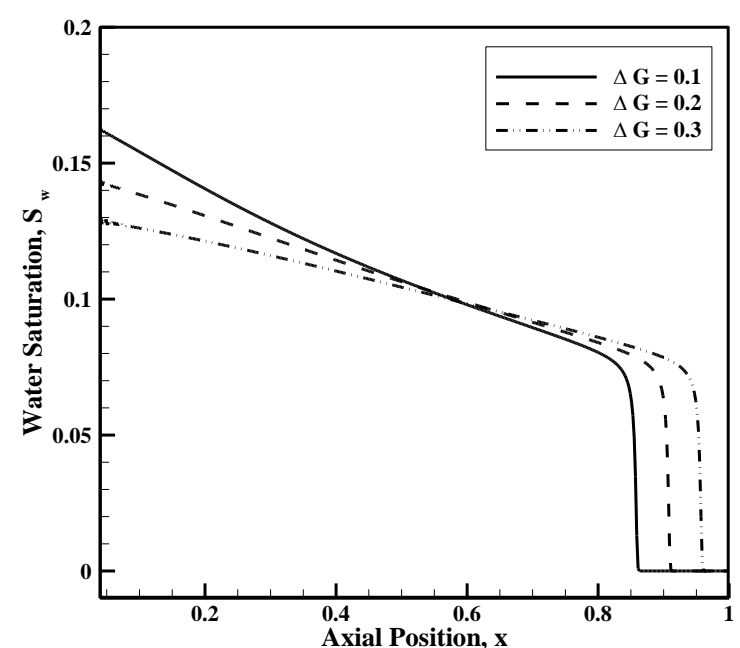

(a)

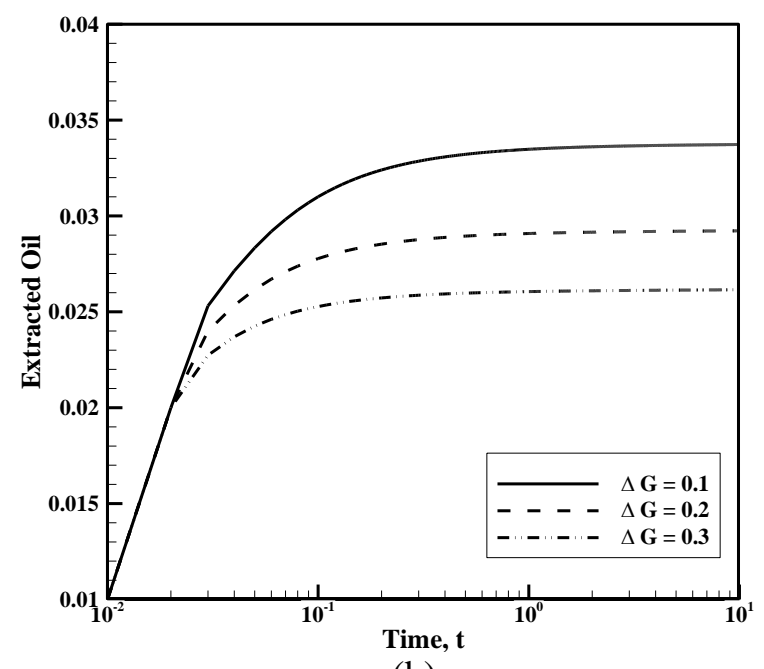

(b)

Figure 9 


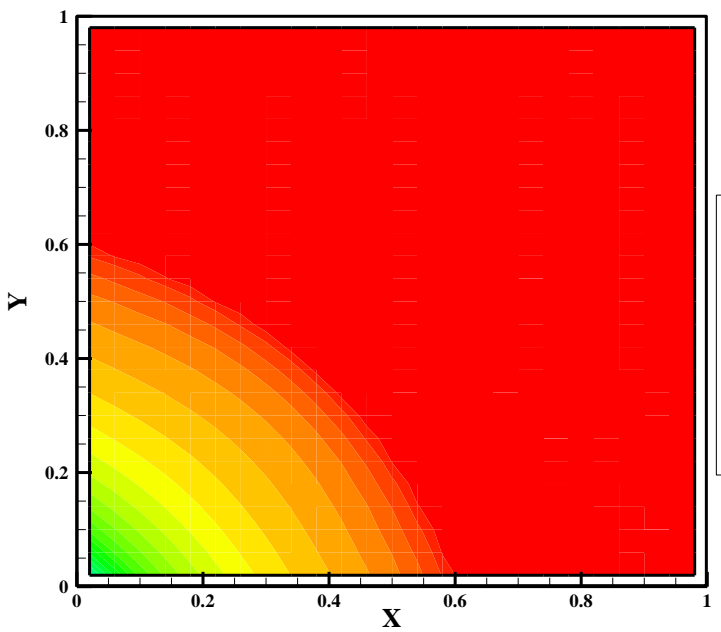

(a)

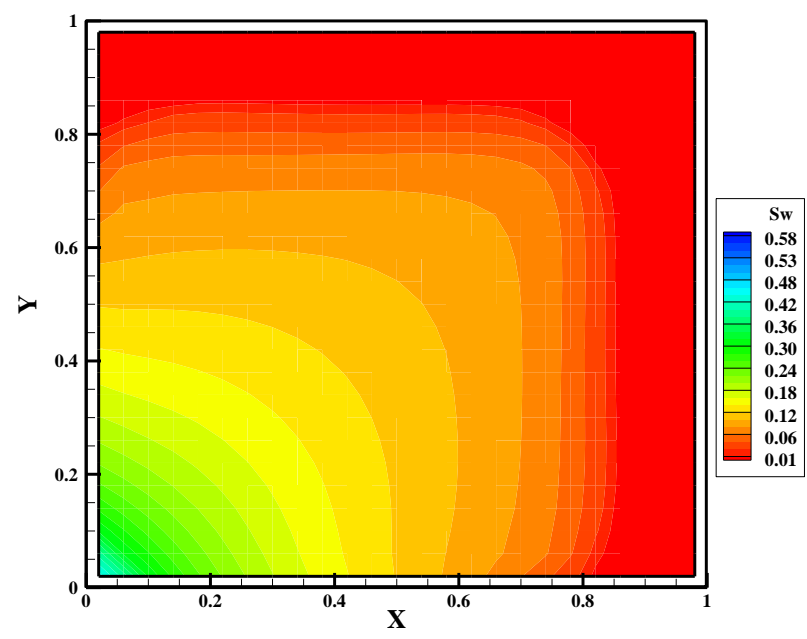

(c)

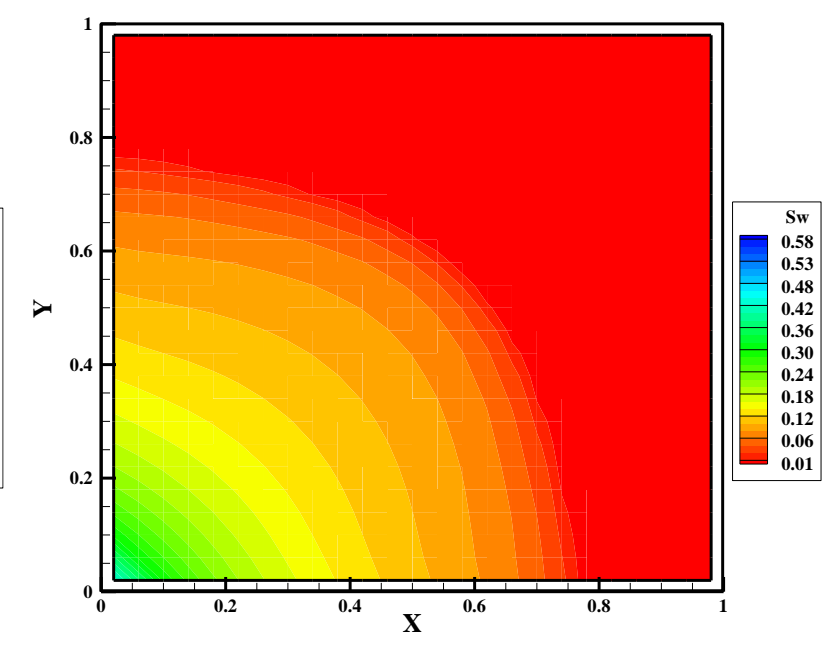

(b)

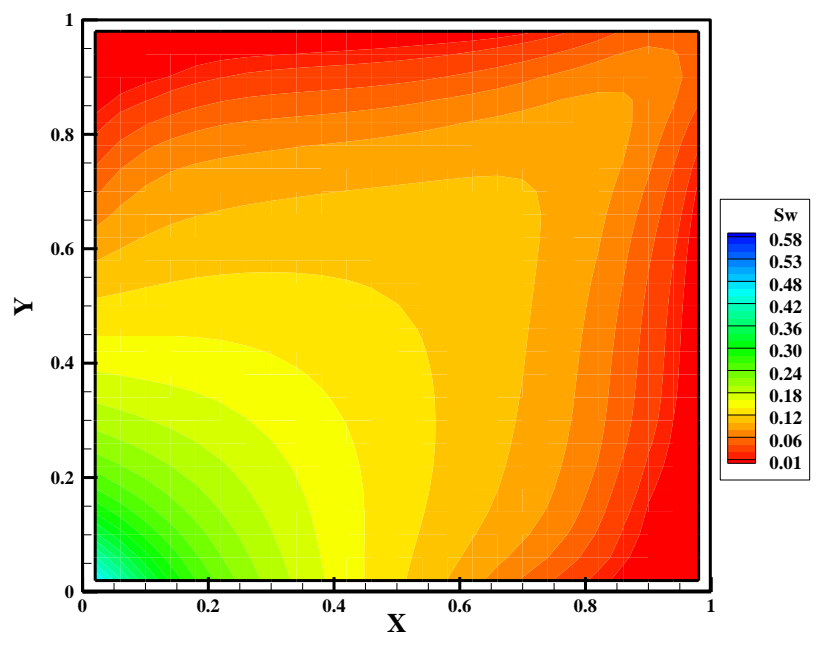

(d)

Figure 10 


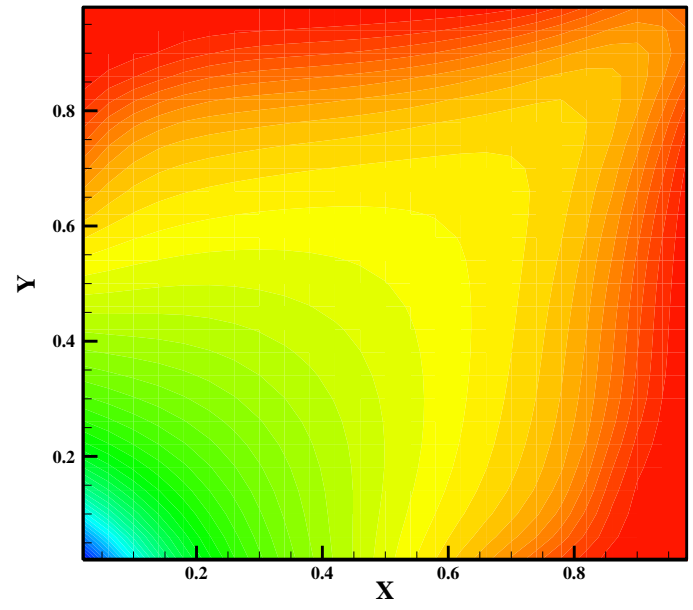

(a)

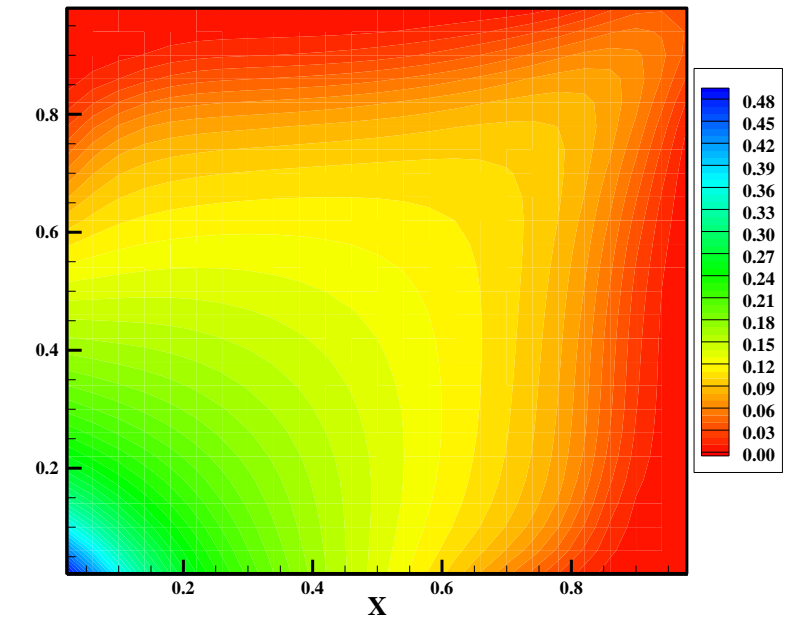

(b)

Figure 11

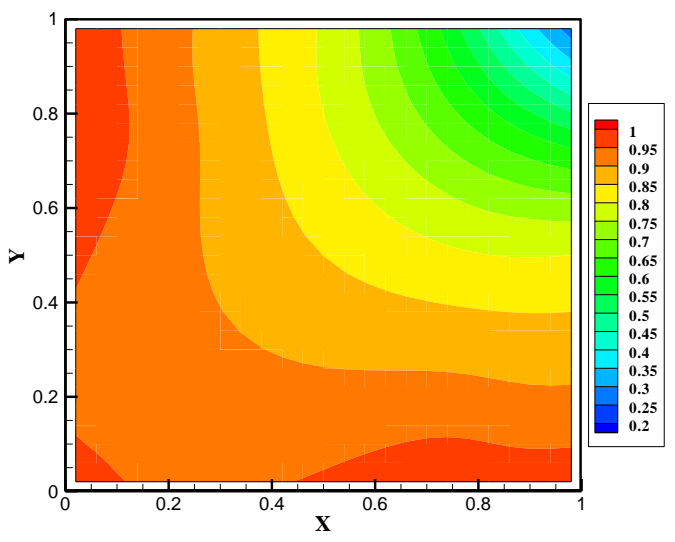

(a)

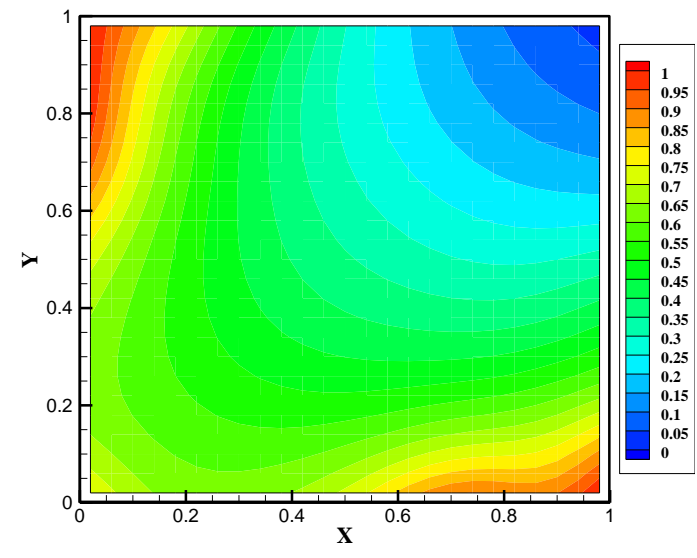

(b)

Figure 12 


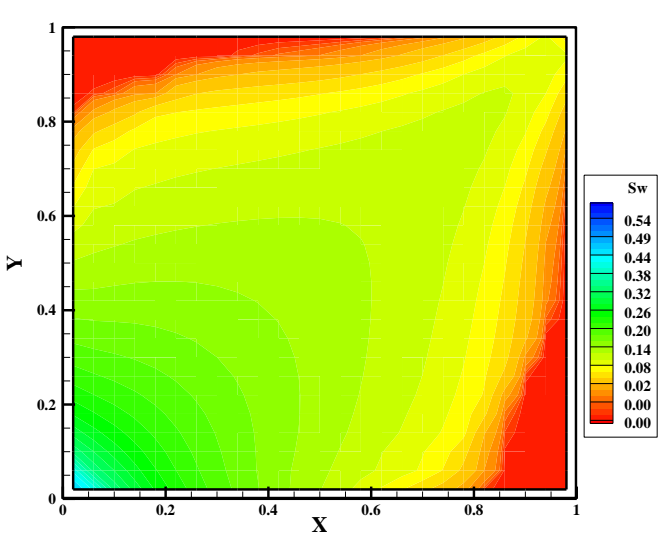

(a)

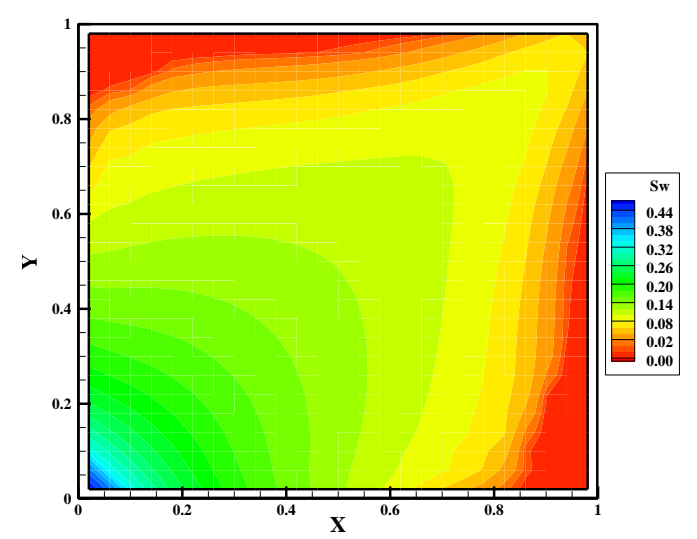

(b)

Figure 13

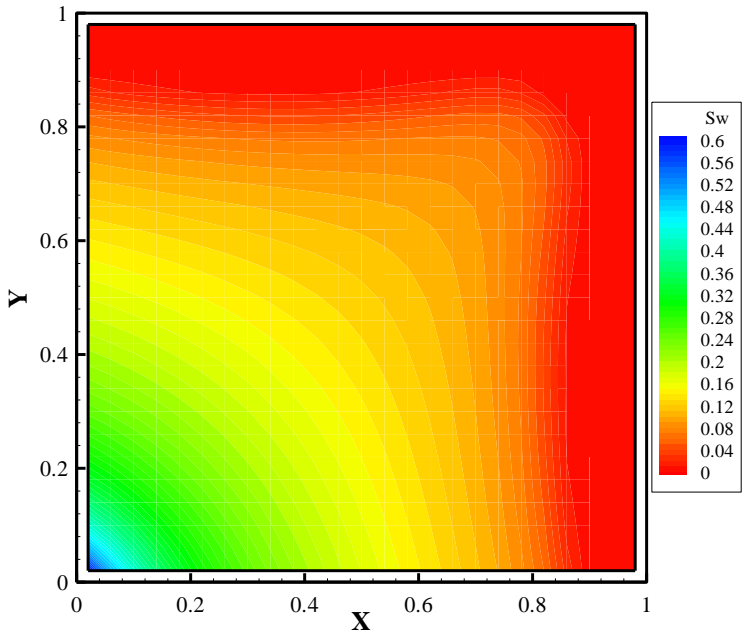

(a)

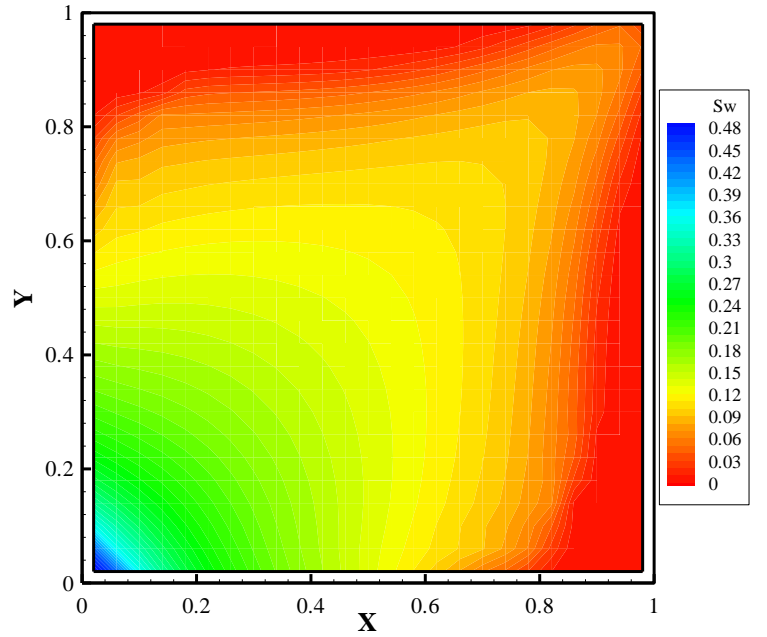

(b)

Figure 14 


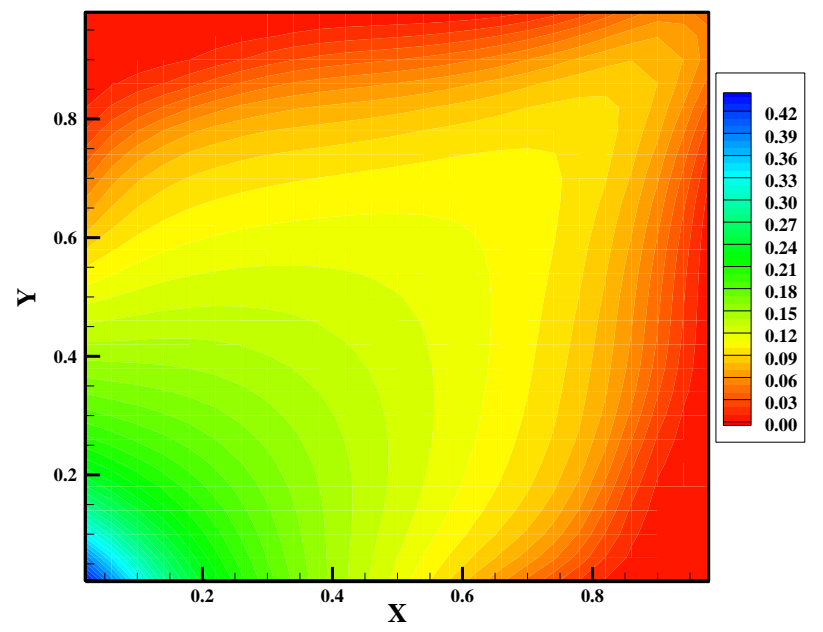

(a)

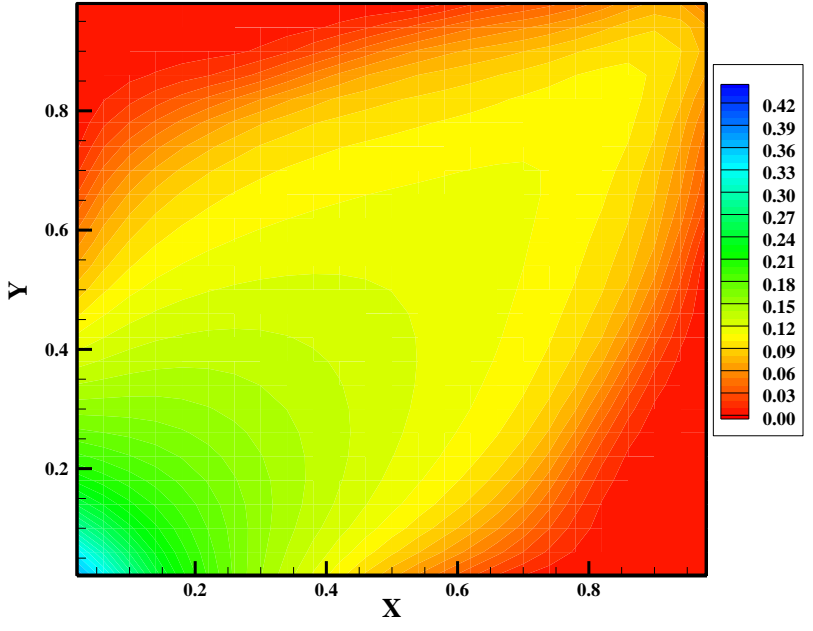

(b)

Figure 15

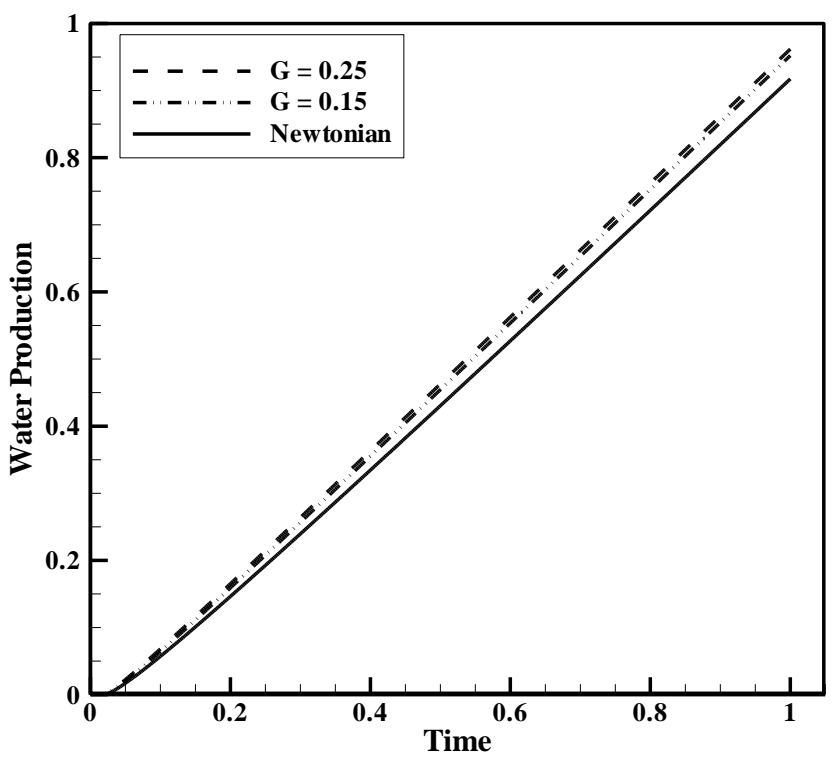

Figure 16 


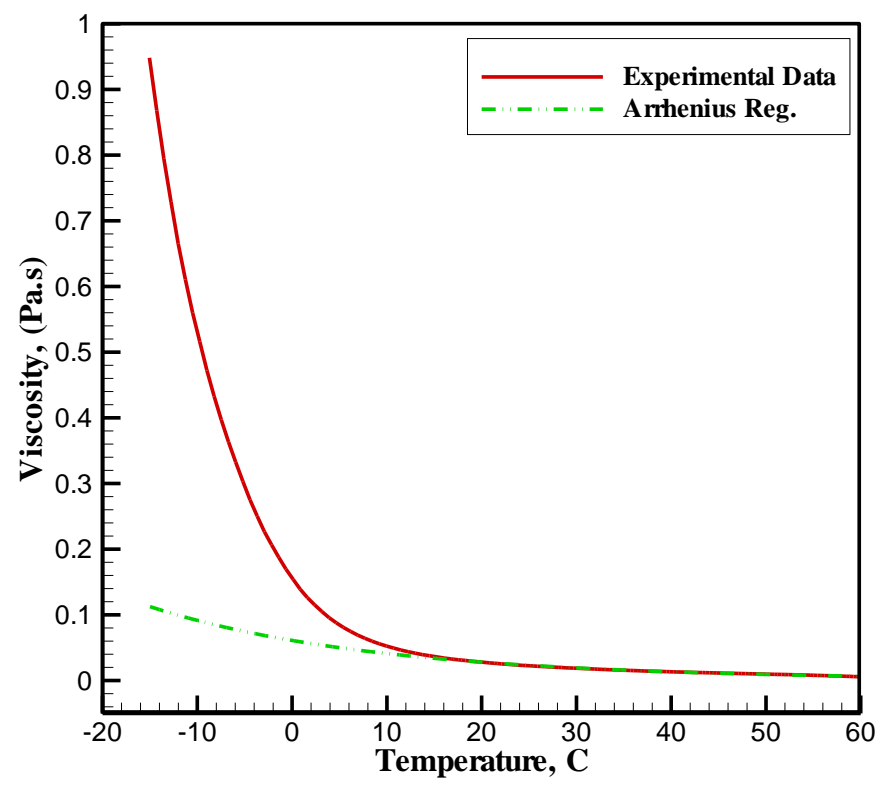

Figure A1

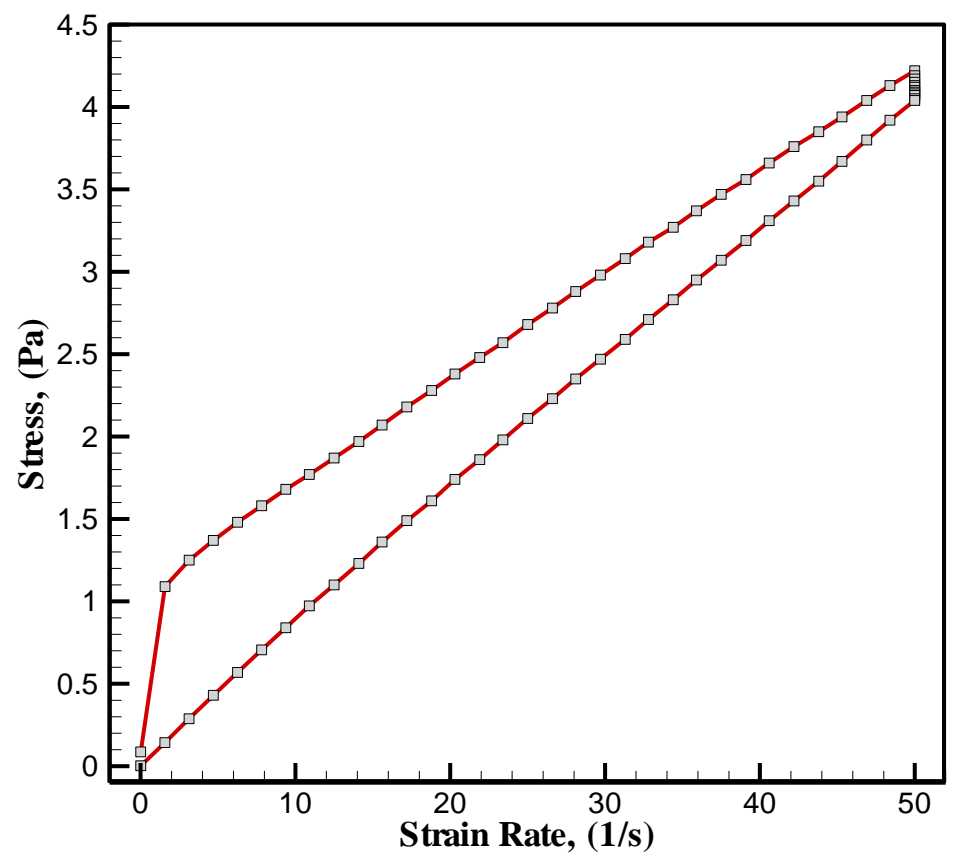

Figure A2 


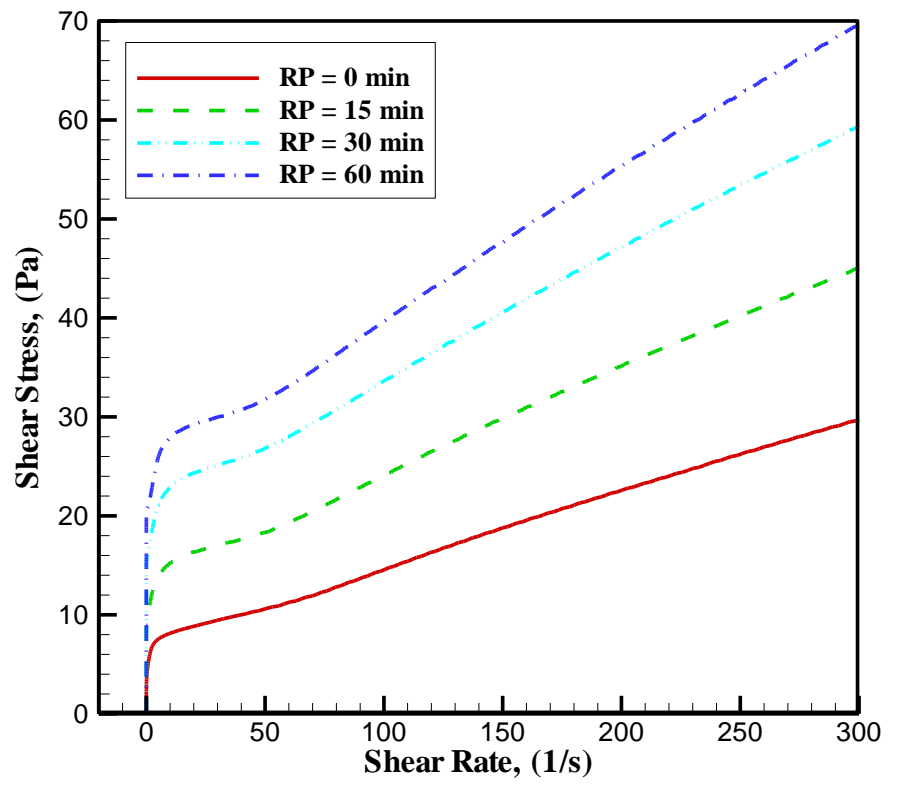

Figure A3

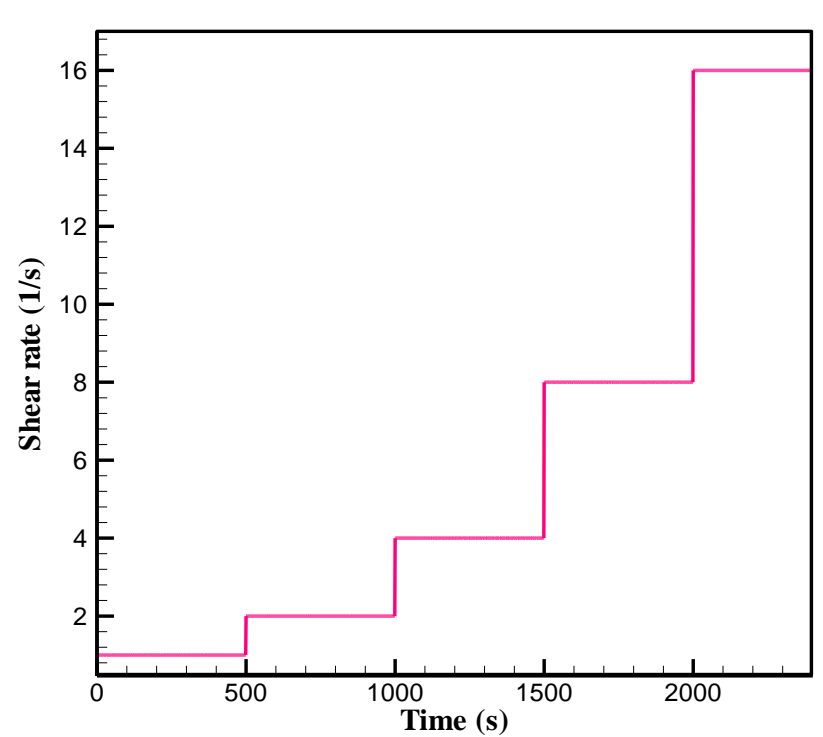

(a)

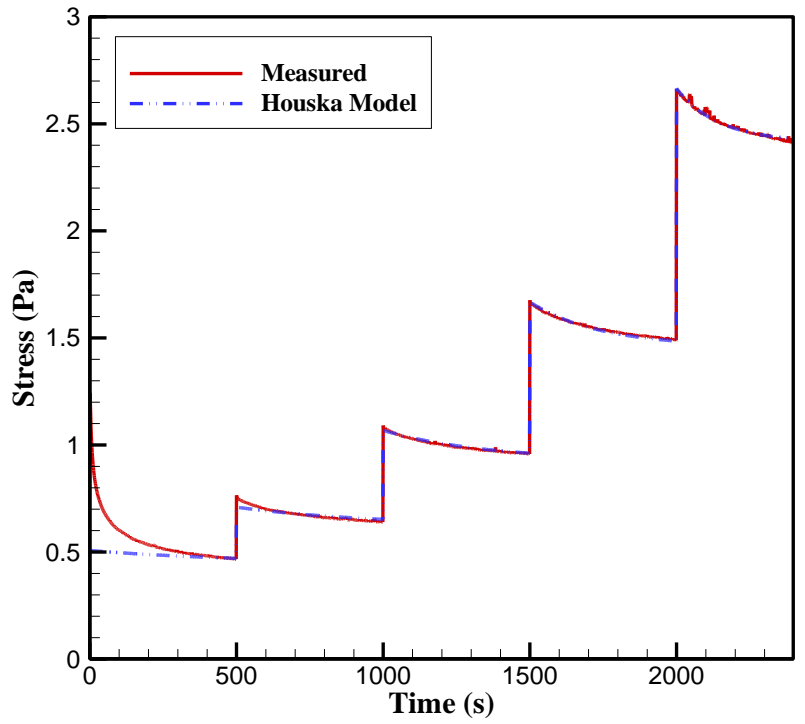

(b)

Figure A4 


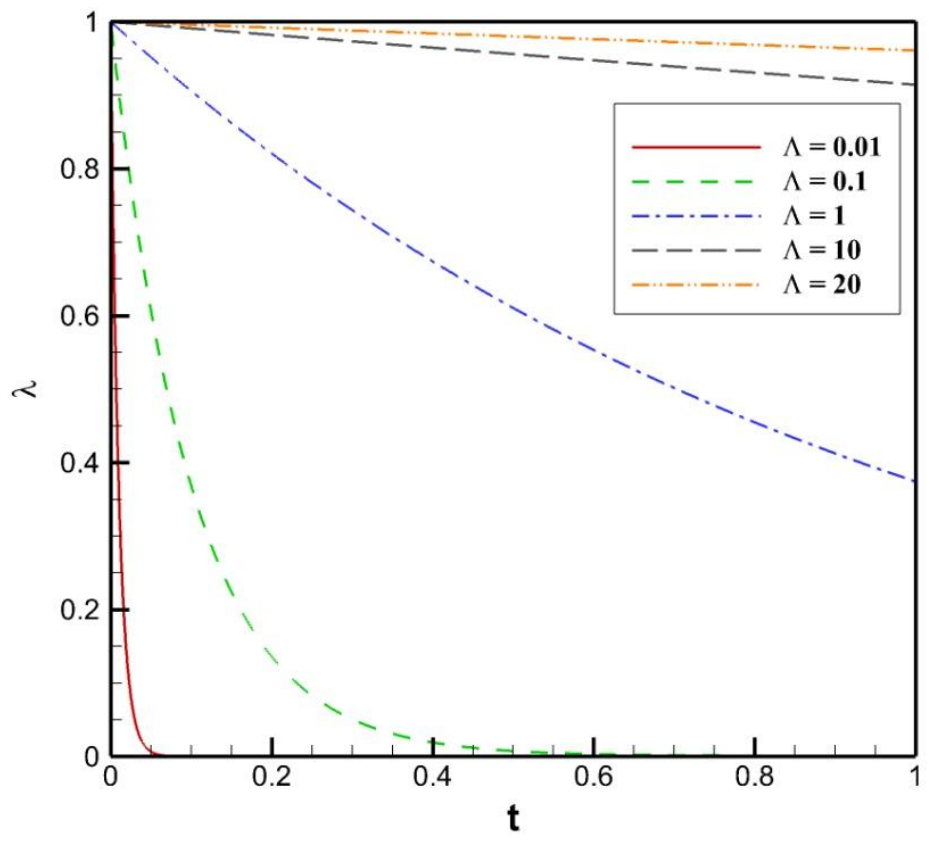

Figure B1

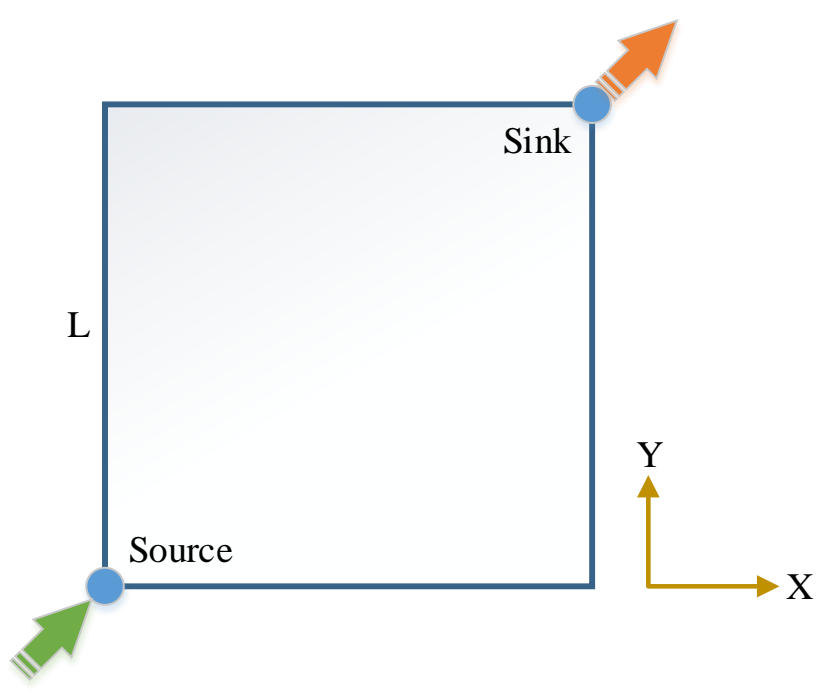

Figure C1 


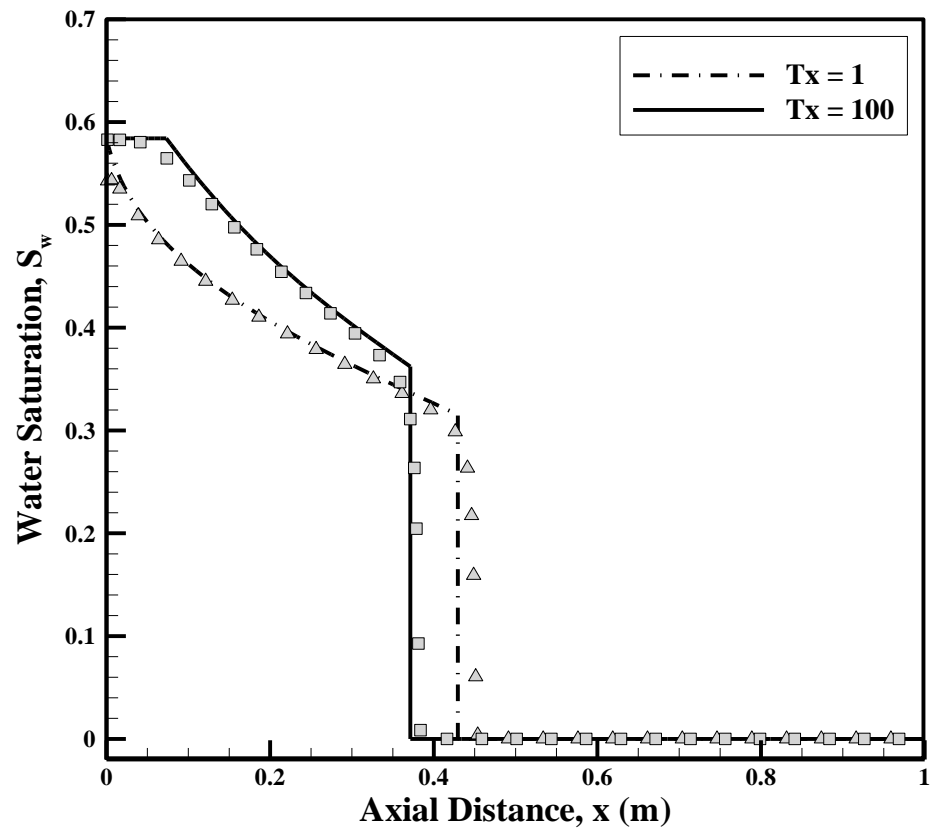

Figure C2 